\title{
The VLA Galactic Plane Survey
}

\author{
J. M. Stil ${ }^{1}$ and A. R. Taylor ${ }^{1}$ \\ J. M. Dickey ${ }^{2,3}$ and D. W. Kavars ${ }^{3}$ \\ P. G. Martin ${ }^{4,5}$, T. A. Rothwell ${ }^{5}$, and A. I. Boothroyd ${ }^{4}$ \\ Felix J. Lockman ${ }^{6}$ \\ N. M. McClure-Griffiths ${ }^{7}$
}

\begin{abstract}
The VLA Galactic Plane Survey (VGPS) is a survey of H I and 21-cm continuum emission in the Galactic plane between longitude $18^{\circ}$ and $67^{\circ}$ with latitude coverage from $|b|<1.3$ to $|b|<2.3$. The survey area was observed with the Very Large Array (VLA) in 990 pointings. Short-spacing information for the $\mathrm{H}$ I line emission was obtained by additional observations with the Green Bank Telescope (GBT). H I spectral line images are presented with a resolution of $1^{\prime} \times 1^{\prime} \times 1.56 \mathrm{~km} \mathrm{~s}^{-1}(\mathrm{FWHM})$ and $\mathrm{rms}$ noise of $2 \mathrm{~K}$ per $0.824 \mathrm{~km} \mathrm{~s}^{-1}$ channel. Continuum images made from channels without $\mathrm{H}$ I line emission have $1^{\prime}$ (FWHM) resolution. The VGPS images reveal structures of atomic hydrogen and 21-cm continuum as large as several degrees with unprecedented resolution in this part of the Galaxy. With the completion of the VGPS, it is now possible for the first time to assess the consistency between arcminute-resolution
\end{abstract}

\footnotetext{
${ }^{1}$ Department of Physics and Astronomy, University of Calgary, 2500 University Drive NW, Calgary, AB, T2N 1N4, Canada

${ }^{2}$ School of Mathematics and Physics - Private Bag 37, University of Tasmania, Hobart, TAS 7001, Australia

${ }^{3}$ Department of Astronomy, University of Minnesota, 116 Church Street, SE, Minneapolis, MN 55455, USA

${ }^{4}$ Canadian Institute for Theoretical Astrophysics, University of Toronto, 60 St. George Street, Toronto ON, M5S 3H8, Canada

${ }^{5}$ Department of Astronomy and Astrophysics, University of Toronto, 60 St. George Street, Toronto ON, M5S 3H8, Canada

${ }^{6}$ National Radio Astronomical Observatory, P.O. Box 2, Green Bank, West Virginia 24944, USA

${ }^{7}$ Australia Telescope National Facility, CSIRO, P.O. Box 76, Epping NSW 1710, Australia
} 
surveys of Galactic H I emission. VGPS images are compared with images from the Canadian Galactic Plane Survey (CGPS) and the Southern Galactic Plane Survey (SGPS). In general, the agreement between these surveys is impressive, considering the differences in instrumentation and image processing techniques used for each survey. The differences between VGPS and CGPS images are small, $\lesssim 6 \mathrm{~K}$ (rms) in channels where the mean $\mathrm{H}$ I brightness temperature in the field exceeds $80 \mathrm{~K}$. A similar degree of consistency is found between the VGPS and SGPS. The agreement we find between arcminute resolution surveys of the Galactic plane is a crucial step towards combining these surveys into a single uniform dataset which covers $90 \%$ of the Galactic disk: the International Galactic Plane Survey (IGPS). The VGPS data will be made available on the World Wide Web through the Canadian Astronomy Data Centre (CADC).

Subject headings: ISM: atoms — Galaxy: disk — Surveys

\section{Introduction}

The physical processes in the feedback cycle of matter between stars and the interstellar medium play an important role in the evolution of galaxies, and in the way products of stellar nucleosynthesis are dispersed. A quantitative description of these processes requires knowledge of the poorly known topology of different phases of the interstellar medium and the timescales and locations of transitions between these phases. Atomic hydrogen is the link between the gas heated or expelled by massive stars and cold molecular gas from which new stars form. $\mathrm{H}$ i is widely distributed and, within certain limits, easily observable across the Galaxy through the 21-cm line. As such, atomic hydrogen has been used to study the dynamics of the Galaxy and physical conditions in the diffuse interstellar medium.

To study the interstellar medium in transition, from the cold phase to the warm phase or vice versa, or the dynamical effect of stars on the interstellar medium, requires observations which reveal parsec-scale structures. For objects outside the solar neighborhood, resolving parsec-scale structures requires arcminute-resolution images. The distribution of cold atomic gas may be revealed by absorption of continuum emission or absorption of the $\mathrm{H}$ I line emission itself (H I self absorption). Results from such observations depend strongly on the resolution of the data (Dickey \& Lockman 1990). To place these processes into a Galactic context, the high resolution image should also show structure on large scales. At $21 \mathrm{~cm}$ wavelength, images with arcminute resolution and a large spatial dynamic range can be obtained by an interferometer in combination with a large single dish telescope to fill in large scale structure not detected by the interferometer. 
Previously the Canadian Galactic Plane Survey (CGPS) (Taylor et al. 2003) and the Southern Galactic Plane Survey (McClure-Griffiths et al. 2001, 2005) have provided highresolution $\mathrm{H}$ I images in the northern sky (mainly the second Galactic quadrant) and the southern sky (third and fourth Galactic quadrants). The CGPS will be extended to longitude $50^{\circ}$, while the SGPS has been extended to longitude $20^{\circ}$. A large part of the first Galactic quadrant is located near the celestial equator. This area of the sky cannot be observed at sufficient angular resolution by the interferometers used in the CGPS and SGPS, because these interferometers have exclusively or mostly east-west baselines. At the extremes of the CGPS and SGPS, the angular resolution of these surveys is degraded by a factor $\sim 3$ in declination.

The Very Large Array (VLA) can observe the equatorial part of the Galactic plane with adequate resolution. In this paper we present the VLA Galactic Plane Survey (VGPS). The VGPS is an H I spectral line and 21-cm continuum survey of a large part of the first Galactic quadrant. This survey was done with the Very Large Array (VLA) and the $100 \mathrm{~m}$ Robert C. Byrd Green Bank Telescope (GBT) of the National Radio Astronomical Observatory (NRAO). Short spacing information for the VGPS continuum images was provided by a continuum survey with the $100 \mathrm{~m}$ Effelsberg telescope (Reich \& Reich 1986; Reich et al. 1990). The CGPS, SGPS, and VGPS will be combined into a single data set which provides arcminute-resolution images of Galactic H I for $90 \%$ of the Galactic plane as part of the International Galactic Plane Survey (IGPS). With the completion of the VGPS, different parts of the IGPS overlap for the first time, allowing a detailed comparison of the results from each survey. This paper describes the VGPS data, and compares the VGPS spectral line images with those of the CGPS and SGPS in the areas where the surveys overlap.

\section{Observations and data reduction}

\subsection{VLA observations}

The main set of observations of this survey was done with the Very Large Array (VLA) of the NRAO. The technical specifications of the array are described in detail by Taylor, Ulvestad \& Perley (2003). The VLA is an interferometer with 27 elements, each $25 \mathrm{~m}$ in diameter (32' FWHM primary beam size at $21 \mathrm{~cm})$. Several VLA fields must be combined in a mosaic to image an appreciable part of the Galaxy. The most compact configuration of VLA antennas, the D-configuration, has baselines between $35 \mathrm{~m}$ and $1.03 \mathrm{~km}$, and is the most suitable for imaging of widespread Galactic H I emission. For short observations (snapshots), the largest angular scale that can be observed reasonably well is $\sim 450$ arcseconds at $21 \mathrm{~cm}$. The synthesized beam size is about 45 arcseconds (FWHM) at $21 \mathrm{~cm}$. The total amount 
of observing time at the VLA allocated to this survey was 260 hours in the period July to September 2000. In addition to the VLA observations, a fast survey with the $100 \mathrm{~m}$ GBT of the NRAO was done to obtain the necessary short-spacing information for the $\mathrm{H}$ I spectral line images. Table 1 lists basic parameters of the VGPS.

\subsubsection{Mosaic Strategy}

An interferometer survey using the mosaicking technique to combine many primary beam areas into a large map begins with the choice of the area to cover and the telescope time available. These two numbers set the overall sensitivity or noise level of the survey, but this sensitivity is not uniform over the area. The gain in a single VLA field varies over the $32^{\prime}$ (FWHM) primary beam of the VLA antennas. When multiple fields are combined in a mosaic, the spacing between pointing centers determines the corrugation or spatial variation of the sensitivity function. A centered hexagonal geometry is optimum for flattening the sensitivity function, for a given number of pointings, but the function can always be flattened further by decreasing the spacing between adjacent beam centers and so increasing the total number of pointings observed. The overhead costs in telescope drive time and the complexity of the data reduction are increased as the number of pointings increases, so there is a compromise required between flattening the sensitivity function and reducing the number of pointings. For this survey we have used the relatively wide spacing of $25^{\prime}$ between points, which is not much less than the full-width to half-power point of the VLA beam at $\lambda 21-\mathrm{cm}$ of $32^{\prime}$. This results in the sensitivity function shown in Figure 1 . The $25^{\prime}$ spacing choice is driven by the 20 second minimum lag time between the end of data taking on one scan and the beginning of the next, which is exacerbated by the need to delete the first 10 second data average of each scan. These features of the current VLA data acquisition system make it a relatively slow telescope for mosaicking. There are other options available for data taking while driving the telescope (mode OF), but these proved to be impractical for this survey.

The D configuration of the VLA is subject to shadowing, i.e. blockage of one antenna by another, at low elevations. For a mosaic survey, it is even more important than usual to avoid shadowing, because of its effect on the primary beam shape of the blocked antenna, which compromises the estimate of the telescope response to a model source that is needed for the maximum entropy deconvolution. This survey includes many fields at negative declinations, for which the available hour angle range is minimal. Avoiding shadowing becomes the first driver of the observing strategy. Figure 2 shows a rough guide for the hour angle and declination range which is safe from shadowing for the $\mathrm{D}$ configuration. All scans for the 
primary survey area $\left(|b|<1^{\circ}, 18^{\circ}<l<65^{\circ}\right)$ were taken without shadowing, as were most in the secondary area $\left(|b|>1^{\circ}\right)$.

Besides avoiding shadowing and minimizing telescope drive times, the most important consideration for scheduling was obtaining multiple scans on each field at widely spaced hour angles. This is the best way to minimize sidelobes due to large unsampled regions of the $u v$ plane that compromise the dynamic range of the resulting maps. The scheduling process was driven by the need to spread the observations of each field over the available hour angle range at the declination of that field, and still fit everything into about 25 sessions, each typically covering $14^{\mathrm{h}}<\mathrm{LST}<24^{\mathrm{h}}$. The low longitude end, which is observable only from $\sim 16^{\mathrm{h}}$ to $\sim 20^{\mathrm{h}}$ LST, was hard to fit into approximately 25 sessions. The area north of $+10^{\circ}$ declination $\left(l>45^{\circ}\right)$ is relatively easy to schedule. For simplification, each row of pointings at constant longitude (six beam areas, 20 minutes of integration time total) was scheduled in a block. The final observing sequence gave hour angle coverages as shown in Figure 3. Top priority for scheduling was given to the primary area of the survey, latitude $-1^{\circ}$ to $+1^{\circ}$, longitude $18^{\circ}$ to $65^{\circ}$. The fields at higher positive and negative latitudes were observed with second priority, so their $u v$ coverage is less evenly distributed over the available hour angle range. Generally the strongest continuum sources that cause the worst dynamic range problems are located in the primary area, so this strategy is appropriate. However it should be kept in mind that the quality of the imaging in the area of the survey with $|b|>1^{\circ}$ is degraded relative to the lower latitudes. Most fields (93\%) were observed at least three times at different hour angles, and $38 \%$ of the fields were observed four or more times. About $7 \%$ of the fields were observed only 2 times. The theoretical sensitivity of the spectral line mosaics is $8.4 \mathrm{mJy}(1 \sigma)$ or $1.4 \mathrm{~K}$ per $0.824 \mathrm{~km} \mathrm{~s}^{-1}$ channel for the beam size of $60^{\prime \prime}$. The rms noise amplitude in the final VGPS images is $1.8 \mathrm{~K}$ per channel on average. The actual spatial variation of the noise in the final VGPS images deviates somewhat from the regular pattern shown in Figure 1 because of differences in the integration time per field. Such differences exist because of variation in the number of visits to a field and because of a longer dwell time on the first field of a block of six.

\subsubsection{Spectrometer}

This survey pushes the limits of the VLA correlator in that we need high resolution in velocity $\left(0.824 \mathrm{~km} \mathrm{~s}^{-1}=3.90 \mathrm{kHz}\right.$ channel spacing $)$ and broad bandwidth $\left(\sim 300 \mathrm{~km} \mathrm{~s}^{-1}\right.$ $=1.4 \mathrm{MHz}$ total). This was impossible to obtain with the existing spectrometer for two polarizations at once. To sacrifice one of the two circular polarizations would be equivalent to giving up half the integration time of the survey, so we chose a strategy that keeps both 
polarizations but with the coarser velocity channel spacing of $1.28 \mathrm{~km} \mathrm{~s}^{-1}$. We then stagger the placement of the channels between the two polarizations by half a channel spacing, $0.64 \mathrm{~km} \mathrm{~s}^{-1}$, so that the sampling on the spectral axis may be increased when all data are combined (Figure 4). The VGPS spectral line data are sampled on the same $0.824 \mathrm{~km} \mathrm{~s}^{-1}$ spectral channels as the CGPS for maximum consistency between the two datasets. The spectral resolution of the data $\left(1.21 \times 1.28 \mathrm{~km} \mathrm{~s}^{-1}=1.56 \mathrm{~km} \mathrm{~s}^{-1}\right)$ is determined by the size of the time lag window in the spectrometer. It is not changed by resampling to narrower spectral channels. The centers of the two polarizations are offset by $+32.304 \mathrm{~km} \mathrm{~s}^{-1}$ and $-31.460 \mathrm{~km} \mathrm{~s}^{-1}$ from the nominal center velocity, which is set at $v_{c}(l)=+80-(1.6 \times l) \mathrm{km} \mathrm{s}^{-1}$ with $l$ the longitude in degrees. Combining these gives spectra with velocity width $341 \mathrm{~km} \mathrm{~s}^{-1}$ after dropping 20 channels on either side of the band due to the baseband filter shape. The $\mathrm{H}$ I line emission is unpolarized except for tiny amounts due to the Zeeman effect, which are far below our sensitivity limit. But to avoid spurious spectral features arising from $\mathrm{H}$ I absorption of linearly polarized continuum, which is common in the synchrotron emission at low latitudes, we alternate the frequency settings between the two polarizations every 100 seconds. So a single observation of a survey field consists of two short integrations (100s each) with complementary spectrometer settings. This gives enough bandwidth to cover the range of velocities in the first Galactic quadrant, +150 to $-80 \mathrm{~km} \mathrm{~s}^{-1}$ at the lower longitudes, with $0.824 \mathrm{~km} \mathrm{~s}^{-1}$ channels throughout. The local oscillator settings for the survey were -3.2 , 3590 with bandwidth code $5(1.5625 \mathrm{MHz}$, of which the inner $\sim 85 \%$ is usable) and correlator mode 2AD. No on-line Hanning smoothing was performed, and the single dish bandpass shape was not used to normalize the spectra, as is sometimes done as part of the correlation step.

\subsection{VLA Calibration}

Calibration of the VLA data was carried out using standard procedures within AIPS. The primary calibrators 3C286 and 3C48 were used for flux and bandpass calibration. After calibration, the visibility data were imported into MIRIAD for further processing. Editing out glitches in the large volume of visibility data for the 990 VLA fields was done with an automated flagging routine. The visibility data for the VGPS fields were searched for highamplitude points relative to the median visibility amplitude for a particular baseline in a particular channel and for a particular spectrometer/polarization combination. The thresholds applied in this procedure were chosen after careful inspection of the data. Amplitudes more than 20 Jy above the median amplitude in the duration of the snapshot were flagged. Also, scans with an overall median amplitude above 50 Jy were flagged to eliminate saturated

antennas. Special care was taken not to label legitimate signal on the shortest baselines as 
bad data. The snapshots are sufficiently short that a constant visibility amplitude can be assumed for each baseline in a single channel in this search.

The flagging procedure allowed streamlined visual inspection of identified bad data and, if necessary, a human veto before the actual flagging. No false rejections were found because the rejection criteria were sufficiently conservative. If visibilities for a particular combination of time, baseline and polarization were rejected in one channel, all channels were rejected so as to keep the $u v$ sampling identical for all channels. Lower amplitude glitches often had higher amplitude counterparts in other channels. The policy to flag all channels was found to be effective in eliminating low-amplitude glitches as well. After the automated flagging procedure, only a few incidental manual flagging operations were required to make spectral line and continuum images free from noticeable effects of bad data.

Preliminary continuum mosaics were constructed from visibility data averaged over channels outside the velocity range of Galactic H I line emission. These mosaics were analyzed with an automated source extraction algorithm to compare the fluxes of compact continuum sources with fluxes in the NVSS survey (Condon et al. 1998). Sources were labeled as suspected variables and removed from consideration if the ratio of the absolute value of the difference between the NVSS and VGPS fluxes and the mean of these fluxes was larger than 10 times the formal error. This comparison between the NVSS and VGPS showed that fluxes of compact sources in the VGPS were on average $30 \%$ less than fluxes listed in the NVSS. The origin of this discrepancy is not understood. It is believed to be the result of the higher system temperature in the VGPS observations, which is in part the result of bright $\mathrm{H}$ I emission in the Galactic plane. The VLA has an automatic gain control (AGC) system that scales the signal with the system temperature, but visibility amplitudes are corrected

for this scaling. First we discuss various factors that affect the system temperature in the VGPS. Later we derive a correction to the flux scale of the VGPS to make it consistent with the NVSS.

\subsubsection{Contributions to the system temperature}

Compared with the NVSS, there are two important enhanced contributions to the system temperature. One contribution is from bright Galactic H I and continuum emission, which can double the system temperature averaged over the inner $75 \%$ of the frequency band. The system temperature changes across the spectral band because the brightness of the $\mathrm{H}$ I line changes with velocity. The other contribution is from spillover to the ground when a field is observed at low elevation. Emission from the atmosphere also depends on elevation, adding 2 to $4 \mathrm{~K}$ to the system temperature. The effect of spillover is an order of magnitude larger than 
this. The average system temperature of the VLA antennas in the zenith is $T_{\text {sys }} \approx 35 \mathrm{~K}$. The system temperature increases rapidly at low elevation, to approximately $70 \mathrm{~K}$ at elevation $30^{\circ}$. VGPS observations were made over a wide range of hour angles to obtain adequate sampling in the $u v$ plane. As a result, fields were regularly observed far from the meridian at low elevation. In contrast, when the NVSS was made, its fields were observed close to the meridian in order to minimize ground noise. This strategy is more suitable for a continuum survey which targets compact sources.

The system temperature for each antenna of the VLA is recorded and stored with the visibility data. However, for the present purpose it is more convenient to adopt a different measure of $T_{\text {sys }}$ derived directly from the visibility data. The scalar average amplitude of the continuum-subtracted visibilities (abbreviated here as ampscalar) gives a spectrum that is proportional to the rms visibility amplitude in each sample; it is noise dominated (proportional to $T_{\text {sys }}$ ). It takes into account the editing of data rejected by the automated flagging routine, and it is not necessarily averaged in frequency as is the system temperature recorded with the data. After continuum subtraction, only the shortest baselines contain some correlated signal because of the Galactic H I line. When averaging the ampscalar data over all antennas, this remaining signal has a negligible effect. This was verified by comparing the result with ampscalar values averaged over baselines longer than $1 k \lambda$. The ampscalar values are proportional to the recorded system temperature averaged over the array.

We write the total system temperature as the sum of the receiver temperature $T_{\text {rec }} \approx$ $35 \mathrm{~K}$, an elevation-dependent term $T_{\text {earth }}(h)$ which includes atmospheric emission but is usually dominated by spillover to the ground, and the brightness temperature of cosmic radio emission $T_{b}(v)$ which depends on velocity because of the bright Galactic $\mathrm{H}$ I line,

$$
T_{\text {sys }}(v, h)=T_{b}(v)+T_{\text {earth }}(h)+T_{\text {rec }}
$$

The spectral line data of the VGPS allows separation of the contribution of Galactic emission to $T_{\text {sys }}$ from other contributions. This in turn allows us to derive a new functional form for the elevation dependence of $T_{\text {earth }}(h)$.

The brightness temperature in each velocity channel, $T_{b}(v)$, averaged over the VLA primary beam, was determined by smoothing the GBT maps and the Effelsberg maps to the resolution of the VLA primary beam. Figure 5 shows the relation between sky brightness temperature (line + continuum) averaged over the primary beam of the VLA, with the scalar averaged amplitude per channel. Three visits to the same field on three different days are shown. The main difference between the snapshots in Figure 5 is the elevation of the field at the time of observation. Some fields were observed at nearly the same elevation on different days. Such observations have nearly indistinguishable values of ampscalar. The relation between ampscalar and brightness temperature was fitted with a linear relation 
to allow extrapolation to $T_{b}=0 \mathrm{~K}$. The contribution of Galactic emission to the system temperature is eliminated by this extrapolation. We refer to this extrapolation as the scalaraveraged amplitude at $T_{b}=0$ or ampscalar at $T_{b}=0$, which is proportional to $T_{\text {earth }}(h)+T_{\text {rec }}$ according to Equation (1). We find that the slope of the relation in Figure 5 increases as the ampscalar at $T_{b}=0$ increases.

Figure 6 shows the relation between the scalar-averaged visibility amplitude and elevation of the field at the time of observation for all snapshots taken on 2000 September 15. A poor correlation is found between the raw band-averaged ampscalar and elevation. After correction for the contribution by Galactic emission, a very tight relation is found between the ampscalar at $T_{b}=0$ and elevation. The scatter in this relation is consistent with the estimated errors in the extrapolation to $T_{b}=0$. This extrapolation is less accurate towards the brightest continuum sources because the relation as shown in Figure 5 is not well defined. The points which do not fit on the relation represent snapshots with very bright continuum. Similar results were obtained for each day of VGPS observations.

Figure 6 illustrates the relative importance of factors which raise the system temperature. At high elevations, Galactic emission roughly doubles the system temperature in the Galactic plane. This increase is mostly due to the bright $\mathrm{H}$ I line, but the continuum also contributes 5 to $20 \mathrm{~K}$ to the system temperature, depending on longitude. The brightest continuum sources (W49, and W51) actually contribute more to the system temperature than the $\mathrm{H}$ I line when averaged over the spectral band.

The tight correlation between ampscalar at $T_{b}=0$ and elevation resembles the increase in $T_{\text {sys }}$ with elevation from spillover to the ground measured by Taylor, Ulvestad \& Perley (2003), who applied a second-order polynomial fit to the data. It is difficult to interpret the results of a polynomial fit when comparing different days of observation, because some days covered more fields at low elevation than others. Inspection of all the data for each day separately showed that the elevation dependence of ampscalar at $T_{b}=0$ is also described well with two free parameters by the form

$$
A=a \cos ^{4} h+b
$$

Equation (2) was fitted to each day and polarization separately. Three fields centered on $(l, b)=(43.3,-0.1),(49.0,-0.5)$, and $(49.4,-0.3)$ were excluded from these fits. These fields are most affected by W49 and W51. Results of the fits are listed in Table 2.

Equation (2) provides an accurate fit of the elevation-dependent noise contribution for each day. Note that the scatter around the fits in Figure 6 is much smaller than the difference between the $\mathrm{L}$ and $\mathrm{R}$ polarization. The mean difference between the two polarizations is $\left\langle b_{L}-b_{R}\right\rangle=0.13 \pm 0.05$. The difference in ampscalar between the polarizations is equivalent 
to a system temperature that is approximately $12 \%$ higher in $\mathrm{L}$ than in $\mathrm{R}$. The rms residuals of the fits $\left(\sigma_{L}\right.$ and $\sigma_{R}$ in Table 2) are 0.06 in the mean. Inspection of the fits indicated that larger values of $\sigma_{L}$ and $\sigma_{R}$ indicate variation in the system temperature during the day. Such variation may be related to solar activity, in particular in September when the angular distance of the Sun was smaller. Inspection of the fits showed that variations in $a_{L}$ and $a_{R}$ in Table 2 appear to be related mainly to intra-day variation. However, the range of values of $b_{L}$ and $b_{R}$ represents real variations in the receiver temperature $T_{r e c}$ during the period of observations.

The fits in Table 2 allow us to make an elevation correction for the noise amplitude for each snapshot to obtain a prediction of the noise level if the field had been observed at the zenith. Figure 7 shows the distribution per VGPS field of the smallest value of the band averaged ampscalar of all snapshots contributing to a field. This map typically shows the ampscalar for the observation of each field at the highest elevation. Most of the structure in the distribution of the band averaged ampscalar corresponds to the VGPS observing sequences of six fields in a row. A few bright continuum sources near the Galactic plane can be identified as well. The observing pattern visible in the distribution of the bandaveraged ampscalar shows that almost everywhere in the survey area elevation-dependent contributions to $T_{\text {sys }}$ are important. Elevation-dependent effects and sky emission contribute roughly equal amounts to the total system temperature (Figure 6).

The minimum ampscalar per field tends to be higher at low longitudes, in part because these fields transit the meridian at a lower elevation. Equation (2) allows a correction to be made for the elevation of the field at the time of observation. The parameters $a$ and $b$ were determined for each day and for each polarization separately by fitting Equation (2) to the available data as illustrated in Figure 6. The elevation-corrected band-averaged ampscalar was calculated by subtracting the excess in the band-averaged ampscalar value resulting from the elevation of the field. The map of the elevation-corrected ampscalar in Figure 7 shows a higher noise level in the Galactic plane, and toward bright continuum sources. Some residuals of the elevation correction remain in this map. These are likely the result of intraday variations that are not traced by the daily average of the elevation correction applied here.

The distribution of the elevation-corrected ampscalar shows a strong resemblance to the VGPS continuum image. This resemblance is not inconsistent with the fact that the $\mathrm{H} \mathrm{I}$ line usually contributes more to the level of $T_{\text {sys }}$ than the continuum. The visualization in Figure 7 tends to emphasize the variations between adjacent fields. Field-to-field variations in the velocity-integrated $\mathrm{H}$ I brightness are fairly small compared with field-to-field variations in the continuum level. 


\subsubsection{Correction of the VGPS flux scale}

The remainder of this section describes a method to adjust the flux scale of the VGPS to the flux scale of the NVSS. The Canadian Galactic Plane Survey also corrects its flux scale to the NVSS by comparing the fluxes of continuum sources in each CGPS field as part of the field registration process described in Taylor et al. (2003). A direct comparison of source fluxes per VGPS field is not possible because, on average, only four compact continuum sources are available per VGPS field. Bright extended continuum emission from H II regions and supernova remnants also inhibits a comparison with NVSS sources. A subset of the VGPS fields contains one or more isolated compact continuum sources that can be compared with corresponding entries in the NVSS catalog. We obtain an empirical relation between the NVSS to VGPS flux ratio and the system temperature, including all contributions, as measured by the band-averaged ampscalar. This relation is used to predict a specific correction factor for each snapshot.

To this end, each continuum snapshot was imaged and cleaned individually. Cleaning VLA snapshot images is difficult because of the high level of sidelobes of the synthesized beam. This is a particular concern for faint sources. Therefore, only sources with a flux density larger than $100 \mathrm{mJy}$ were considered. Sources identified in the snapshot images were matched with sources in the NVSS catalog. A source was accepted if its position was within $15^{\prime \prime}$ of the NVSS position, and if the deconvolved size of the source in the NVSS catalog was less than $60^{\prime \prime}$. These requirements are aimed to avoid misidentification while retaining a sufficient number of sources to determine a relationship between the band averaged ampscalar and the flux ratio NVSS/VGPS.

The snapshot images were sorted into narrow ranges of the band averaged ampscalar. For each narrow range, a list of identified sources was compiled. Each of these source lists contains continuum sources throughout the VGPS survey area detected in snapshots with nearly the same band-averaged ampscalar. Figure 8 shows the ratio of NVSS flux to VGPS flux as a function of the band averaged ampscalar. A tight relation is found between the band averaged ampscalar and the flux ratio NVSS/VGPS. This relation defines the correction factor for the flux scale of each snapshot based on its band averaged ampscalar. The scatter in the relation shown in Figure 8 is dominated by source number statistics. The effect of the sample size was investigated by regenerating the relation for fifty randomly selected subsamples, each half the size of the total number of snapshots. The error bars in Figure 8 show the rms variation for each bin over the fifty subsamples. Values of the band-averaged ampscalar are always larger than 1.4 in the VGPS. A second-order polynomial was fitted to the data to provide a prescription for the correction factor, as a first-order polynomial fit was deemed insufficient. A small number of fields that include very strong continuum 
emission have a band averaged ampscalar outside the range where the relation is defined. We assume the relation to be constant for band averaged ampscalar more than 3.8. All VGPS continuum mosaics were made again with this correction to the flux scale. These new mosaics were searched for compact continuum sources and the fluxes of these sources were compared with fluxes listed in the NVSS catalog. This comparison included many faint sources that were not included in the derivation of the flux correction. The flux scale of the VGPS mosaics was found to be consistent with the NVSS flux scale within $5 \%$.

The success of applying the well-defined relation in Figure 8 adds confidence to the initial assumption that the difference in the flux scale between VGPS and NVSS continuum images was related to the higher system temperature in the VGPS. However, the negative slope in this relation implies that the largest correction to the flux scale is necessary for VGPS snapshots with the lowest system temperature. This result remains unexplained. However, we note that increased contributions to the system temperature by bright continuum emission and by spillover to the ground at smaller Galactic longitudes must also have occurred to some extent in the NVSS observations.

The response of the VLA to increased system temperature has been tested by following a flux calibrator during a time span of several hours as it approaches the horizon. These experiments indicate that the raw correlator output is adequately corrected for the scaling of the signal by the AGC (R. A. Perley, private communication). Calibrators observed for the VGPS are not well suited to repeat this experiment because they cover a very limited range in elevation. In particular, the primary flux calibrators were consistently observed at high elevation. With limited significance, we confirmed that the raw correlator output for the secondary calibrators did not change with elevation. Our analysis shows that a calibrator must be followed to very low elevations to probe the system temperature regime of VGPS observations. The behavior of ampscalar at $T_{b}=0$ in Figure 6 is representative for a calibrator, because the $T_{b}(v)$ term in Equation $(1)$ is very small for a calibrator. A comparison of the two panels of Figure 6 shows that the system temperature in VGPS target fields almost always exceeds the system temperature of a calibrator at an elevation of only $25^{\circ}$. However, observations of a calibrator as it sets to elevations as low as $10^{\circ}$ (Taylor, Ulvestad \& Perley 2003) cover the system temperature range of most VGPS observations.

The relation in Figure 8 compares flux measurements of fairly bright, relatively isolated, compact continuum sources. These flux measurements should not be affected by modest differences in the $u v$ coverage between the VGPS and the NVSS. Since ampscalar values do not change if the minimum baseline is taken as long as $1 k \lambda$, the ampscalar values are also not affected by differences in $u v$ coverage. Therefore, we believe that the behavior in Figure 8 is not related to differences in $u v$ coverage between the VGPS and the NVSS. 
In summary, the VGPS calibration follows these steps:

1. Standard gain and phase calibration was done in AIPS for all snapshots. The gain and phase corrections were applied to the data before importing the visibilities into MIRIAD for imaging.

2. Visibilities were channel-averaged (continuum images) or continuum-subtracted (spectral line images) as appropriate.

3. The gain for each snapshot was adjusted by a single factor derived from its band averaged ampscalar and the relation shown in Figure 8. A small number of fields was selected for self-calibration after construction of the mosaics (Section 2.4.1).

\subsection{GBT observations}

Short-spacing information for the $\mathrm{H}$ I line emission was obtained using the Green Bank Telescope (GBT) of the National Radio Astronomy Observatory (NRAO). The GBT is a $100 \mathrm{~m}$ dish with an off-axis feed arm for an unblocked aperture which reduces the radio sidelobes, radio frequency interference, spectral standing waves, and the effects of stray radiation. The extent of the GBT survey varies with longitude, covering $|b| \leq 1.3$ for longitudes $18^{\circ} \leq l \leq 45^{\circ}$ and $|b| \leq 2.3$ for longitudes $45^{\circ} \leq l \leq 67^{\circ}$. Observations of these regions began on 2002 November 21-25 and continued on 2003 March 6-9, 2003 May 26-27, and 2003 August 29-30. The observing strategy was to make small, $\Delta l=2^{\circ}-5^{\circ}$, H I maps 'on the fly.' Using this technique, the telescope was driven at a rate of $3^{\circ}$ per minute with a sample written every second. After driving through the full latitude range, the telescope was stepped $3^{\prime}$ in longitude. This process was continued until the particular map was completed. The data were taken in frequency-switching mode using the GBT spectral processor with a total bandwidth of $5 \mathrm{MHz}$ across 1024 channels. The resulting channel spacing is $1.03 \mathrm{~km} \mathrm{~s}^{-1}$ and the spectral resolution is $1.25 \mathrm{~km} \mathrm{~s}^{-1}$ (FWHM). Data were taken by in-band frequency switching yielding a total velocity coverage of $530 \mathrm{~km} \mathrm{~s}^{-1}$ centered at $+50 \mathrm{~km} \mathrm{~s}^{-1} \mathrm{LSR}$. IAU standard regions S6 and S8 (Williams 1973) were observed and used for absolute brightness temperature calibration. The angular resolution of the GBT data is $\sim 9^{\prime}$. The final GBT $\mathrm{H}$ I spectra have an $\mathrm{rms}$ noise of $\sim 0.3 \mathrm{~K}$ in emission-free channels.

Imaging and data calibration were done with the AIPS ++ data reduction package. A first order polynomial was fitted to the off-line channels to remove residual instrumental baseline structure. Polynomial fits of higher order were also attempted, but these were found to be no better than a first order polynomial fit. Gridding of the data was done with the task IMAGEMS, using a BOX gridding function. The latitude and longitude size of each cell was 
set to $3^{\prime}$, equal to the longitude spacing of the observations. Each of the small H I maps had a narrow overlap in longitude with the adjacent maps. After applying the absolute brightness temperature calibration, the overlap regions were compared for consistency. A majority of the overlap regions showed small discrepancies of order $1-3 \mathrm{~K}$, but a few regions were found to have brightness temperature differences of up to 6-8 K. The overlap regions were used to scale the cubes observed on different days to a common calibration, which is consistent to within $3 \%$. As a second check of the observed brightness temperature consistency, a small portion of the galactic plane $\left(65^{\circ} \leq l \leq 67^{\circ} ;-1.3^{\circ} \leq b \leq+1.3^{\circ}\right)$ was observed twice, once during the 2002 November 21-25 observing session and again during the 2003 August 29-30 session. Only small differences of a few Kelvin were found between these maps.

\subsection{Imaging}

The definition of VGPS mosaic images is a direct extension of the set of mosaic images of the CGPS. VGPS images are sampled in position and velocity on the same grid as CGPS images. Mosaics of $1024 \times 1024$ pixels $\left(5.12 \times 5^{\circ} .12\right)$ are centered at intervals of $4^{\circ}$ in longitude. This provides significant overlap between mosaics for coverage of objects near the mosaic boundary. The velocity axis of VGPS spectral line cubes is sampled with the same channel definitions as the spectral line cubes of the CGPS, but the velocity range covered by the VGPS is different. There are three VGPS data products as described below: continuum images which include short-spacing data, continuum-subtracted H I spectral line images which include short-spacing data, and continuum-included spectral line images which do not include short-spacing data. All VGPS data products will be made available on the World Wide Web through the Canadian Astronomy Data Centre (CADC).

\subsubsection{Continuum}

VGPS line or continuum images are mosaics of several VLA pointings. As it is impractical to process the entire survey area at the same time, only fields with their central longitudes within $4^{\circ}$ of the center of the mosaic were included in the construction of each mosaic. Each VLA field was imaged to the $10 \%$ level of the primary beam, and field images were combined with the VLA primary beam as weighting function. The primary beam model for the VLA is the same as used in the AIPS task LTESS.

Figure 9 shows the sampling in the $u v$ plane and the synthesized beam for a representative field near the center of the survey, composed of snapshots taken at three different hour 
angles. A Gaussian weighting function in the $u v$ plane was applied to obtain a $60^{\prime \prime}$ (FWHM) synthesized beam throughout the survey area. The strongest sidelobes of the synthesized beam are spokes running radially outward from the main lobe in several position angles. The pattern of the spokes depends on the hour angles at which the field was observed. The pattern is different for each field, but fields that were observed within the same sequence of six fields (Section 2.1), have similar but slightly rotated patterns. The amplitude of the spokes in the dirty beam is typically $10 \%$ of the main lobe, but peaks up to $17 \%$ are usually present. The VGPS images must be deconvolved to remove artifacts resulting from these sidelobes. Emission in the Galactic plane usually fills the field of view. In this case, deconvolution is preferred after mosaicking the fields.

Continuum images were constructed from visibility data averaged over channels without discernible line emission, but avoiding noisy channels near the edges of the frequency band. Deconvolution of the VGPS images was done with the MIRIAD program MOSMEM which uses the maximum entropy method described by Cornwell \& Evans (1985) and Sault et al. (1996). Experiments showed that no significant improvement was made after about 20 iterations of the algorithm, even if no formal convergence could be reached. A maximum of 50 iterations was adopted in the deconvolution of the continuum mosaics. The criteria that define the convergence of the deconvolution, the entropy function and the $\chi^{2}$ criterium, include a summation over the entire image. Therefore, the result of the deconvolution depends somewhat on the area that was imaged in the construction of the mosaic. This causes small differences between neighboring mosaics in the area where they overlap, with rms amplitude at or below the level of the noise.

Standard calibration alone results in images with a maximal dynamic range of $\sim 100$, which is not sufficient to image bright continuum sources in the survey area without discernible image artifacts. The continuum mosaics were inspected for residual sidelobes around bright sources after the deconvolution step. Self-calibration was attempted for selected fields with strong artifacts, beginning with the field in which the source is located closest to the field center. A small mosaic, typically including all fields within $\sim 1^{\circ}$ of the source, was made and deconvolved. If a particular visit to the field could be identified as the prime origin of the artifacts, the visibility data from this visit were excluded from the initial imaging step. The deconvolution of this small mosaic produces a sky model of "clean components", which is used for phase self-calibration on the central field only, after multiplication with the primary beam model of the VLA. The small mosaic is then made with the improved calibration solution for the central field. If any data were left out of the first imaging step, those data were included after the first round of self calibration. If necessary, adjacent fields are self-calibrated subsequently. The central field is usually self-calibrated a second time after self-calibration of the surrounding fields. After self-calibration, the final VLA continuum 
mosaic was constructed and regridded to Galactic coordinates.

The results of the self-calibration are usually satisfactory. A dynamic range of $\sim 200$ was obtained in the continuum images after self calibration on sources that mostly affect a single field. However, self-calibration does not provide a good solution if the source is too far from the field center. The brightest continuum sources in the VGPS, in particular W49 and W51, generate artifacts even at large distances from the field center. These artifacts remain in the continuum images, mostly affecting fields surrounding these sources.

The VLA is not sensitive to emission on angular scales larger than $\sim 30^{\prime}$ because structures on these scales are resolved even by the shortest projected baselines. The missing continuum short-spacing information was provided by the continuum survey of Reich \& Reich (1986) and Reich et al. (1990) with the $100 \mathrm{~m}$ Effelsberg telescope. The deconvolved VLA mosaic was combined with the Effelsberg image with the MIRIAD program IMMERGE. The interferometer and single-dish images are both Fourier transformed and added in the $u v$ plane with baseline-dependent weights such that the sum represents the visibilities for all angular scales weighted by a single Gaussian weighting function defined by the $1^{\prime}$ synthesized beam. More details of this procedure were given by McClure-Griffiths et al. (2005). The single-dish data may be multiplied with a factor close to 1 to compensate for a potential difference in the flux calibration between the two surveys. This factor can be determined by comparing the Fourier transforms of the single-dish and interferometer images in a common annulus

in the $u v$ plane, taking into account the difference in resolution. This flux scale factor was found to be equal to 1 within the uncertainties of a few percent, so it was set to 1 for all VGPS continuum mosaics.

\subsubsection{H I spectral line}

Continuum emission was subtracted in the $u v$ plane by subtracting the average of visibilities in the channels used to create the continuum images. The continuum-subtracted visibility data were resampled from their original $1.28 \mathrm{~km} \mathrm{~s}^{-1}$ channels to $0.824 \mathrm{~km} \mathrm{~s}^{-1}$ channels before imaging with the velocity resampling method included in MIRIAD. The resampled visibility data in an output channel are the weighted mean of visibilities of all input channels that overlap with the output channel. The weights are proportional to the amount of overlap between each input velocity channel with the output velocity channel. VGPS velocity channels were defined to match with velocity channels of the CGPS. The resulting velocity sampling was tested by comparing VGPS continuum absorption profiles with corresponding profiles from other sources, in particular the CGPS. These tests showed that the strategy of staggering velocity channels (Figure 4) and subsequent resampling of 
the data in MIRIAD was successful in recovering a fully-sampled $\mathrm{H}$ i line profile over the required wide velocity range. The resampled visibility data were imaged and mosaicked in the same way as the continuum images.

Continuum sources in absorption appear as a negative imprint in the $\mathrm{H}$ I image in the form of the continuum source convolved with the dirty beam. A continuum source brighter than $T_{\mathrm{b}} \approx 20 \mathrm{~K}$ displays visible sidelobes in channels where the optical depth of the $\mathrm{H} \mathrm{I}$ line is significantly more than 1 . The VGPS area contains many brighter continuum sources which display significant sidelobes in absorption, even if the optical depth of the $\mathrm{H}$ I line is not that high. The maximum entropy deconvolution would consider the sidelobes associated with absorbed continuum sources as structure in the $\mathrm{H}$ I emission and try to deconvolve these structures.

Before deconvolution of the $\mathrm{H}$ I line emission, the sidelobes of bright continuum sources are removed using the clean (Högbom 1974) algorithm with the MIRIAD program MOSSDI. A temporary mosaic with (projected) baselines longer than $0.3 \mathrm{k} \lambda$ was made for this purpose. The exclusion of short baselines in this mosaic eliminates most $\mathrm{H}$ I emission and allows proper cleaning of (negative) absorbed continuum sources without interference from negative sidelobes associated with $\mathrm{H}$ I emission. The resulting clean component model for absorbed sources was subtracted from the H I image and restored with a Gaussian beam before deconvolution of the line emission. This procedure is effective in removing sidelobes associated with absorbed continuum sources, in particular the radial spokes that occur in the synthesized beam of VLA snapshots (Figure 9). The H I line emission was then deconvolved with the same maximum entropy algorithm as used for the continuum images. The deconvolved model of "clean components" of the H I emission was restored with a 1' (FWHM) circular Gaussian beam. The synthesized beam in the VGPS H I images is therefore independent of position and identical to the synthesized beam in the continuum images.

The clean $\mathrm{H}$ I images were regridded to Galactic coordinates and combined with the $\mathrm{H} \mathrm{I}$ zero spacing data set obtained with the GBT. The process of combining the single-dish survey with the interferometer survey allows rescaling of the single-dish data to match the flux scale of the interferometer. The accuracy with which this scale factor can be determined from the H I spectral line data was estimated at $5 \%$ to $10 \%$. Within the uncertainties, no scaling of the GBT flux scale was required. However, after a detailed comparison of $\mathrm{H}$ I images from the VGPS and the CGPS (see Section 3.1), it was decided to rescale the GBT data with a factor 0.973 to obtain a single consistent calibration for the CGPS and the VGPS. This correction factor is well within the uncertainty of the absolute flux calibration. A consistent flux scale for these surveys facilitates the comparison of properties of $\mathrm{H}$ I emission in the inner and the outer Galaxy. 


\subsubsection{Continuum-included $\mathrm{H}$ I cubes}

H I cubes were also constructed with both line and continuum emission included, for use in 21-cm absorption line studies. Instead of subtracting the continuum (as was done for the continuum-subtracted line cubes described in the previous section), the visibility data were imaged directly with the same velocity channels as the spectral line cubes. The weighting function in the $u v$ plane was superuniform weighting, which optimizes the angular resolution of the images. Maximum angular resolution is more important for continuum absorption experiments than brightness sensitivity. A "dirty" continuum-included mosaic cube was created, and deconvolved using the maximum entropy method; the deconvolved model of "clean components" was restored with a 50" circular Gaussian beam (slightly smaller than the 60 " Gaussian beam used to restore the line cubes and continuum maps). The deconvolved cubes were then regridded to Galactic coordinates. Note that no zero-spacing data were added to these continuum-included H I cubes, since no zero-spacing data set was available that included both line and continuum emission.

\section{Comparing the VGPS with CGPS and SGPS}

The three individual Galactic plane surveys will be combined into a uniform high-fidelity multi-wavelength dataset which covers the Galactic plane from $l=-107^{\circ}$ to $l=+190^{\circ}$ at least within the latitude range $|b|<1^{\circ}$ with arcminute angular resolution. This combined dataset is called the International Galactic Plane Survey (IGPS). It will cover 90\% of the area of the Galactic disk. With the completion of the VGPS, different IGPS components overlap in narrow portions of the Galactic plane. This allows for the first time a comparison of these large mosaicking surveys of the H I sky. The success of an integrated IGPS dataset depends on the extent to which the different surveys can produce consistent images when observing the same part of the sky. This is a significant challenge given the different telescopes and imaging techniques used, in particular for the $\mathrm{H}$ I line where extended bright emission is present throughout the field of view. Comparing the VGPS with the CGPS is of particular interest because the image deconvolution step does not exist in the CGPS.

\subsection{VGPS and CGPS}

The CGPS and the VGPS are each a combination of two separate surveys. Low spatial frequencies are sampled by a single-dish survey, and high spatial frequencies are sampled by an interferometer survey. The single-dish survey dominates the signal at spatial frequencies 
smaller than those sampled by the shortest projected baselines of the interferometer. Most of the power in Galactic H I emission occurs on these large angular scales. The low-resolution survey for the CGPS was done with the DRAO 26 meter telescope. The low-resolution survey for the VGPS was done with the $100 \mathrm{~m}$ GBT. These telescopes have different resolution and stray radiation characteristics. Stray radiation correction was done for the low-resolution DRAO survey (Higgs \& Tapping 2000), but not for the GBT survey, as the sidelobes of the GBT are much lower.

The shortest (unprojected) baseline of the DRAO synthesis telescope is $60 \lambda$, compared to $170 \lambda$ for the VLA. The largest angular scale sampled by the interferometer is therefore approximately 3 times larger in the CGPS. On average, the VLA D-array also samples smaller angular scales than the DRAO ST. The longest baselines of the VLA D-array are $\sim 50 \%$ longer than the longest baseline of the DRAO ST. At the declination of the area of overlap $\left(\delta \lesssim 30^{\circ}\right)$ the array configurations and difference in latitude between DRAO and the VLA further increase the difference between the interferometers in projected baselines.

The most significant difference between the VGPS and the CGPS is the density of the sampling of the aperture plane by the interferometer. A full synthesis with the DRAO ST samples the aperture plane completely between an inner and an outer boundary (Landecker et al. 2000). This complete sampling allows direct imaging of the visibility data without deconvolution. The sampling of the aperture plane by VGPS snapshots is less complete. The necessary deconvolution of the VGPS images is a priori the most likely source of differences between CGPS and VGPS H I images.

In order to compare the CGPS and VGPS spectral line data, a special VGPS spectral line cube was made with a resolution that matches the $122^{\prime \prime} \times 58^{\prime \prime}$ synthesized beam of the DRAO ST in the overlap area. The major axis of the DRAO synthesized beam changes by $17 \%$ across the area where the VGPS and CGPS overlap. Assuming an average beam shape for the comparison leads to a maximum mismatch of the beam size of less than $9 \%$ in the declination direction in the extreme corners of the area where VGPS and CGPS overlap. The spectral resolution of the CGPS is $1.32 \mathrm{~km} \mathrm{~s}^{-1}$ (FWHM), marginally smaller than the $1.56 \mathrm{~km} \mathrm{~s}^{-1}$ (FWHM) resolution of the VGPS. The difference is less than half a frequency channel. This small difference in velocity resolution is not expected to produce a noticeable difference because of the modest dynamic range of the spectral line data.

Figure 10 compares H I line profiles from the CGPS and the VGPS. The CGPS and VGPS H I emission profiles agree very well, but comparing $\mathrm{H}$ I emission profiles is not a sensitive test of the consistency for small-scale structure, because the signal is dominated by the single-dish surveys. Line profiles affected by continuum absorption are an exception, because the depth of the absorption profile is very sensitive to resolution. Figure 10 therefore 
focuses on continuum absorption profiles.

Judging the significance of the differences between the two datasets involves the noise in the images at the resolution of $2^{\prime} \times 1^{\prime}$. The rms noise in channels free of line emission in the overlap region, avoiding noisy edges of the CGPS mosaic, is $1.8 \mathrm{~K}$ for the CGPS, and $1.2 \mathrm{~K}$ for the VGPS. These noise amplitudes are consistent with the theoretical noise levels in DRAO spectral line images at the declination of the overlap region (Landecker et al. 2000), and for VGPS data convolved to the $2^{\prime} \times 1^{\prime}$ resolution of the DRAO images. The theoretical off-line noise in the difference profile is therefore $2.2 \mathrm{~K}$ (rms). In channels where the $\mathrm{H}$ I brightness is high $(\sim 100 \mathrm{~K})$, the noise is approximately twice the off-line noise (see Section 2.2). Differences between the H I profiles should therefore be compared to an expected rms noise amplitude $4.4 \mathrm{~K}$. The measured rms amplitude of the difference image per channel is $2.2 \mathrm{~K}$ per velocity channel in the absence of line emission (as expected), and $5.8 \mathrm{~K}$ in channels where the $\mathrm{H}$ I emission is brightest. The rms amplitude of the difference image over the overlap area is $\sim 30 \%$ higher than the theoretical noise amplitude of the difference image. Locally, the differences in Figure 10 are usually less than $10 \mathrm{~K}$, but occasional differences as large as $\pm 20 \mathrm{~K}(4$ to $5 \sigma)$ exist.

Figure 11 shows the difference VGPS-CGPS in a single velocity channel at $+9 \mathrm{~km} \mathrm{~s}^{-1}$. Inspection of the difference images shows that residuals on small angular scales usually coincide with emission features in the original images. Some ripple-like artifacts in the highest rows of VGPS fields $\left(b= \pm 2^{\circ}\right)$ are the result of imperfect convergence of the maximum entropy deconvolution (e.g., Figure 11 near $l=66.25, b=-2^{\circ}$ ). Differences associated with mainly small-scale $\mathrm{H}$ I emission are also believed to originate from the deconvolution. In rare cases these differences appear as a measurable velocity difference between features seen in the CGPS and in the VGPS. However, an analysis of narrow continuum absorption features (Figure 10) showed that in general the velocities in the surveys agree very well.

However, the most conspicuous structure in the difference image is a wave pattern with a wavelength of order 1.5 which does not resemble any structure in the $\mathrm{H}$ I emission. The pattern is seen with the same phase and orientation in channels where the average brightness of the $\mathrm{HI}$ line is high, but its amplitude varies with the brightness of the $\mathrm{H}$ I line. The pattern may be isolated by filtering in the Fourier domain. Subtracting the wave pattern in Figure 10 decreases the rms residuals to $5.2 \mathrm{~K}$. This is more than the expected value of $4.4 \mathrm{~K}$. If the remaining difference is entirely the result of the image deconvolution, then the inconsistencies because of the image deconvolution are less than $3 \mathrm{~K}$ (rms). The crests of the wave pattern are aligned with the field centers of the CGPS or possibly the direction of right ascension and the wavelength corresponds within the errors with the separation of the CGPS fields. The wavelength of the pattern is more than three times the field separation of 
the VGPS. This excludes the VLA data as a possible origin. Also, the zero-spacing datasets of the CGPS and VGPS were taken with a primary beam of $30^{\prime}$ and $9^{\prime}$, excluding a possible problem with the primary beam of these telescopes as the origin. Both zerospacing datasets were observed scanning across the Galactic plane at constant longitude. It is not likely this observing pattern would produce a wave pattern with the orientation seen in Figure 11. This leaves the DRAO ST as the most likely origin of the wave pattern.

The sign of the fluctuations in Figure 11 suggests that the CGPS intensity is systematically higher at larger distances from the field center in the declination direction. This could indicate that the primary beam of the DRAO ST is slightly elliptical, with a larger size in the declination direction than predicted by the axially symmetric model adopted in the mosaicking (Taylor et al. 2003). This hypothesis was confirmed by a reanalysis of point sources fluxes measured for the CGPS field registration (Taylor et al. 2003) provided by S. J. Gibson. In the field registration process, fluxes of compact continuum sources are measured in individual CGPS fields and compared with fluxes listed in the NVSS. This process has accumulated $\sim 17,000$ flux measurements over the years of observations for the CGPS. This dataset was analyzed for systematic changes in the ratio of CGPS flux to NVSS flux as a function of position angle in the field. It was found that the flux ratio CGPS/NVSS was on average a few percent higher along the declination axis. This systematic effect can be removed by applying a slightly elliptical primary beam model for the DRAO ST, with axial ratio $0.975 \pm 0.005$. This value is within the uncertainties of a previous determination of the primary beam shape of individual DRAO antennas (Dougherty 1995), but the comparison with the VGPS shows that it has a significant effect in wide-field imaging of the Galactic H I line.

This comparison shows that despite the critical deconvolution step in the VGPS image processing, the VGPS images are in general very consistent with CGPS images which did not require image deconvolution. We estimated that inconsistencies in the convergence of the VGPS image deconvolution result in differences less than $\sim 3 \mathrm{~K}$ (rms). We have found evidence for a small ellipticity in the primary beam of the DRAO ST, which creates a measurable effect in wide-field images of bright Galactic H I emission. It should be noted that the overlap area between the CGPS and the VGPS is in a part of the sky which is most favorable for the VGPS.

\subsection{VGPS and SGPS}

The Southern Galactic Plane Survey (SGPS) covers a large piece of the Galactic plane in the southern hemisphere between $l=-107^{\circ}$ and $l=+20^{\circ}$ and $|b|<1^{\circ}$. The interferometer 
used is the ATNF Australia Telescope Compact Array (ATCA). Zero spacing data is provided by the multi-beam $64 \mathrm{~m}$ Parkes telescope. The ATCA is mainly an east-west array but it has a north-south arm which was used for the northernmost part of the SGPS (phase II), including the overlap region with the VGPS, to obtain more complete sampling in the $u v$ plane. The ATCA elements are similar in size to the VLA antennas, so individual SGPS fields are similar in size to VGPS fields. Mosaicking speed is improved at the expense of continuity of antenna tracks in the $u v$ plane, in particular at longer baselines. The density of sampling of the $u v$ plane by the ATCA decreases faster with baseline length than for the VLA. The longer baselines of the ATCA are included in SGPS datasets used to study continuum absorption, but not in the $\mathrm{H}$ I spectral line cubes. This limits the resolution of the SGPS H i spectral line images to $\sim 2^{\prime}$ (FWHM). The SGPS and VGPS use the same software for mosaicking and image deconvolution, but there are some differences. The SGPS data acquisition and image processing was described by McClure-Griffiths et al. (2001) and McClure-Griffiths et al. (2005).

The area of overlap between the VGPS and SGPS is smaller than the overlap between VGPS and CGPS. Also, SGPS images have $3^{\prime} \times 2^{\prime}$ resolution in the overlap area, compared to $2^{\prime} \times 1^{\prime}$ for the CGPS. The shape of the synthesized beam of the SGPS changes rapidly across the overlap area. This is relevant for comparing absorption profiles, because continuum sources and their associated absorption are not cleaned separately in the SGPS. Absorbed continuum sources are convolved with the local dirty beam which may have a different shape than the global restoring beam adopted for the line emission. The comparison between SGPS and VGPS images presented here is therefore less detailed. However, this comparison is useful because it is done in the part of the IGPS survey area which is most difficult to observe from the point of view of both the VGPS and the SGPS. It is therefore a comparison of images made under the least favorable conditions.

Figure 12 compares SGPS and VGPS line profiles with the same spatial resolution at two locations. The upper panel shows a representative $\mathrm{H}$ I emission line profile. The correspondence between the VGPS and SGPS profiles is generally as good as between the CGPS and VGPS. The bottom panel in Figure 12 shows a continuum absorption profile. Here, the quality of the correspondence between the profiles depends critically on our ability to match the beam of the SGPS at the location of the source. For comparison, the fullresolution VGPS profile is also shown.

It is found that the VGPS and SGPS H I images (not shown) are consistent to a similar degree as the consistency between the CGPS and VGPS. The comparison between VGPS and SGPS is somewhat limited because of the smaller area of overlap and the varying beam of the SGPS data. 


\section{Panoramic images of the Galactic plane}

The high-resolution images of the VGPS open the opportunity for a variety of research on the interstellar medium in the first Galactic quadrant. The images also reveal objects which would not be noticed in previous surveys with lower resolution. Stil et al. (2004) reported the discovery of a small H I shell in the inner Galaxy. The upper limit to any thermal emission from this shell derived from the VGPS continuum image, imposed strong constraints on the interpretation of this shell as a stellar wind bubble. Lockman \& Stil (2003) and Stil et al. (2006) reported the discovery of small H I clouds with large forbidden velocities from the VGPS data. These clouds with masses of tens of solar masses and radii of a few parsec may be the disk analogy of similar clouds seen at large distances from the Galactic plane (Lockman 2002). Stil et al. (2006) demonstrate the importance of arcminute-resolution images in the discovery of these clouds.

The VGPS data are presented in Figure 13 as panoramic images of the Galactic plane in 21-cm continuum and $\mathrm{H}$ I line emission. The continuum images display a strong increase in complexity of structure as Galactic longitude decreases, reflecting the higher rate of star formation in the inner Galaxy. At lower longitudes, the Galactic plane is traced by a diffuse layer of emission approximately $1^{\circ}$ thick centered on $b=0^{\circ}$. This layer may be the thin disk identified at $408 \mathrm{MHz}$ by Beuermann et al. (1985). A large number of shells, filaments and center-filled diffuse emission regions is seen concentrated toward the Galactic plane, with occasional objects located a degree or more from $b=0^{\circ}$. Many compact continuum sources are also present. At longitudes $l \gtrsim 55^{\circ}$, the Galactic plane is much less discernible in the continuum images. Some small diffuse emission regions and numerous point sources are seen on top of an extended otherwise featureless background of continuum emission. The structures in the VGPS continuum images, with angular scales ranging from several degrees down to the resolution limit of the VGPS, represent processes that operate on a wide range in physical size, time scale, and energy. Many unresolved sources are distant radio galaxies, but some compact Galactic H II regions and supernova remnants may also appear unresolved in the VGPS.

The brightest continuum sources in the VGPS are W49 $\left(l=43^{\circ}\right)$ and W51 $\left(l=49^{\circ}\right)$. The VGPS continuum images contain artifacts within a distance $\sim 1^{\circ}$ around these sources. The dynamic range is limited because self-calibration for a source far from the field center is not possible (see Section 2.4.1). This affects mainly fields surrounding the bright source. Sidelobes of a bright continuum source outside the survey area are present at $l \approx 35^{\circ} .5$, $b \approx-1.3$.

The bottom panels in Figure 13 show $\mathrm{H}$ I emission at $+3.5 \mathrm{~km} \mathrm{~s}^{-1}$. H I at this velocity arises predominantly near the solar circle (Galactocentric radius $8.5 \mathrm{kpc}$ ), and thus both 
locally and from gas 6.6 to $16 \mathrm{kpc}$ distant, depending on longitude. The VGPS covers only a small fraction of the scale height of local gas, but at the far side the latitude range $b= \pm 1.3$ spans more than 700 pc (almost twice the FWHM thickness of the H I layer) perpendicular to the Galactic plane at $l=20^{\circ}$. Any tendency of $\mathrm{H}$ I emission to be concentrated toward the Galactic plane is therefore most likely the result of emission on the far side. At longitudes $l \lesssim 30^{\circ}, \mathrm{H}$ I emission in Figure 13 is brighter around $b=0^{\circ}$ than at $b= \pm 1.3$. Here we see distant $\mathrm{H}$ I at the far side on the solar circle through local H I. This distinction disappears for $l \gtrsim 30^{\circ}$ because the FWHM thickness of the H I layer on the far side becomes comparable to or larger than the latitude range covered by the VGPS.

The local H I is widely distributed in latitude, but it is far from featureless. Most of the structure in the local $\mathrm{H}$ I is difficult to separate from structure in emission from the far side, but there are exceptions. A band of decreased $\mathrm{H}$ I brightness temperature runs approximately perpendicular to the Galactic plane between $l=20^{\circ}$ and $l=22^{\circ}$. This dark band is part of a large cloud of cold H I discovered by Heeschen (1955) and mapped by Riegel \& Crutcher (1972) seen in 'self-absorption' against brighter background H I emission. H I self-absorption features may represent a transition or a boundary between molecular and atomic hydrogen. The high-resolution VGPS images reveal delicate substructure in this large cloud of cold hydrogen. Many H I self-absorption features are seen in VGPS images at positive velocities on a wide range of angular scales.

Measurements of the opacity of continuum absorption provide an estimate of the spin temperature of $\mathrm{H}$ I and therefore the thermal structure of the gas. Continuum absorption profiles can also help resolve the near-far distance ambiguity of kinematic distances for sources of continuum emission in the inner Galaxy. Many resolved and unresolved continuum sources are seen in absorption in the $\mathrm{H}$ I images. These appear as a negative imprint of the continuum image onto the $\mathrm{H}$ I images in Figure 13. However, this imprint is not a perfect copy of the continuum image. A good example is the area between $l=18^{\circ}$ and $l=20^{\circ}$, where some continuum sources are seen in absorption, whereas other sources of similar brightness are not. This clearly illustrates the potential of the VGPS data to study the location and the properties of cold H I clouds in the Galaxy.

Any structural resemblance between $\mathrm{H}$ I emission and continuum emission would be unusual. Continuum emission originates mainly from ionized gas or relativistic electrons. The 21-cm line of $\mathrm{H}$ I traces neutral atomic gas. Apart from the negative imprint of the continuum image because of continuum absorption, there is almost no similarity between the $\mathrm{H}$ I and continuum emission in Figure 13. A peculiar exception is a thin filament of continuum emission at $l=38^{\circ} .35,0^{\circ} \leq b \leq 0{ }^{\circ}$. An $\mathrm{H}$ I filament with the same thickness and center line is observed in the VGPS. It is visible in Figure 13, but the velocity of the 
$\mathrm{H}$ I filament is actually near $0 \mathrm{~km} \mathrm{~s}^{-1}$, where it is much more conspicuous against the other emission.

\section{Conclusions}

$\mathrm{H}$ I spectral line and 21-cm continuum images of the Galactic plane between $l=18^{\circ}$ and $l=67^{\circ}$ from the VLA Galactic Plane Survey (VGPS) are presented. The VGPS data will be made available on the World Wide Web through the Canadian Astronomy Data Centre $(\mathrm{CADC})$.

The calibration of the VGPS images was made consistent with the calibration of the NVSS (Condon et al. 1998). Initially fluxes of compact sources in VGPS images were found to be systematically below the fluxes listed in the NVSS by up to $30 \%$. A correction to the flux scale of the VGPS was developed, based on the noise amplitude in the visibility data. This procedure can be applied to any VGPS field, including those without sufficient point sources to compare the flux directly with the NVSS, and those fields which are filled with complex extended emission. After this correction, the fluxes of compact continuum sources in the VGPS were found to be consistent with NVSS fluxes to within $5 \%$.

VGPS H I images were compared with those from the CGPS and SGPS in regions of overlap and show good agreement between the three surveys, although the rms amplitude of the difference image (VGPS-CGPS) is approximately 30\% higher than the fluctuations expected from the theoretical noise levels alone. Small systematic differences between the VGPS and CGPS originate from imperfections of the image deconvolution applied in the VGPS image processing, and from a wave pattern which resembles the effect of ellipticity in the primary beam of the DRAO ST.

\section{Acknowledgements}

The National Radio Astronomy Observatory is a facility of the National Science Foundation operated under cooperative agreement by Associated Universities, Inc. The VGPS is supported by a grant to A.R.T. from the Natural Sciences and Engineering Council of Canada. This research was supported in part by the National Science Foundation through grants AST 97-32695 and AST 03-07603 to the University of Minnesota. JMS thanks Richard Gooch for adding code to the KARMA software which reads miriad $u v$ datasets used in our automated flagging program, and Steven J. Gibson for providing the CGPS field registration data. The authors thank the anonymous referee for useful comments on the manuscript. 


\section{REFERENCES}

Beuermann, K., Kanbach, G., and Berkhuijsen, E. M. 1985, A\&A, 153, 17

Condon, J. J., Cotton, W. D., Greisen, E. W., Yin, Q. F., Perley, R. A., Taylor, G. B., \& Broderick, J. J. 1998, AJ, 115, 1693

Cornwell, T. J., \& Evans, K. F. 1985, A\&A, 143, 77

Dickey, J. M., \& Lockman, F. J. 1990, ARA\&A, 28, 215

Dougherty, S. M. 1995, DRAO technical report, October 1995

Heeschen, D. S. 1955, ApJ, 121, 569

Higgs, L. A., \& Tapping, K. F. 2000, AJ, 120, 2471

Hogbom, J. A. 1974, A\&AS, 15, 417

Landecker, T. 1., Dewdney, P. E., Burgess, T. A., Gray, A. D., Higgs, L. A., Hoffmann, A. P., Hovey, G. J., Karpa, D. R., Lacey, J. D., Prowse, N., Purton. C. R., Roger R. S., Willis, A. G., Wyslouzil, W., Routledge, D., \& Vaneldik, J. F. 2000, A\&AS, 145, 509

Lockman, F. J. 2002, ApJ, 580, L47

Lockman, F. J., \& Stil, J. M. 2004, in ASP Conf. Ser. 317, Milky Way Surveys: The Structure and Evolution of Our Galaxy, ed. D. Clemens, R. Y. Shah, \& T. Brainerd, (San Francisco: ASP), 20

McClure-Griffiths, N. M., Green A. J., Dickey J. M., Gaensler, B. M., Haynes, R. F., \& Wieringa, M. H. 2001, ApJ, 551, 394

McClure-Griffiths, N. M., Dickey, J. M., Gaensler, B. M., Green, A. J., Haverkorn, M., \& Strasser, S. 2005, ApJS, 158, 178

Reich, P.,\& Reich, W. 1986, A\&AS, 63, 205

Reich, W., Reich, P., \& Fürst 1990, A\&AS, 83, 539

Riegel, K. W., \& Crutcher, R. M. 1972, A\&A, 18, 55

Sault, R. J., Staveley-Smith, L., \& Brouw, W. N. 1996, A\&AS, 120, 375

Stil, J. M., Taylor, A. R., Martin, P. G., Rothwell, T. A., Dickey, J. M., McClure-Griffiths, N. M. 2004, ApJ, 608, 297 
Stil, J. M., Lockman, F.J., Taylor, A. R., Dickey, J. M., Kavars, D. W., Martin, P. G., Rothwell, T. A., Boothroyd, A. I., McClure-Griffiths, N. M. 2006, ApJ, 637, 366

Taylor, A. R., Stil, J. M., Dickey, J. M., McClure-Griffiths, N. M., Martin, P. G., Rothwell, T., Lockman, F. J. 2002, in ASP Conf. Ser. 276, Seeing Through The Dust: The Detection Of H iAnd The Exploration Of The ISM In Galaxies, ed. A. R. Taylor, T. L. Landecker, \& A. G. Wills (San Francisco: ASP), 68

Taylor, A. R., Gibson, S. J., Peracaula, M., Martin, P. G., Landecker, T. L., Brunt, C. M., Dewdney, P. E., Dougherty, S. M., Gray, A. D., Higgs, L. A., Kerton, C. R., Knee, L. B. G., Kothes, R., Purton, C. R., Uyaniker, B., Wallace, B. J., Willis, A. G., \& Durand, D. 2003, AJ, 125, 3145

Taylor, G. B., Ulvestad, J. S., \& Perley, R. A. 2003, on-line document: The Very Large Array Observational Status Summary, 26 May 2003, http://www.vla.nrao.edu/astro/guides/vlas/current/vlas.html

Williams, D. R. W. 1973, A\&AS, 8, 505 
Table 1. VGPS parameters

\begin{tabular}{lrr}
\hline \hline \multicolumn{1}{c}{ Quantity } & \multicolumn{1}{c}{ Value } \\
\hline Survey area & $|b|<1.3$ & $18^{\circ}<l<46^{\circ}$ \\
& $|b|<1.9$ & $46^{\circ}<l<59^{\circ}$ \\
& $|b|<2.3$ & $59^{\circ}<l<67^{\circ}$ \\
Angular resolution (FWHM) & & $60^{\prime \prime} \times 60^{\prime \prime}$ \\
Spectral resolution (FWHM) & $1.56 \mathrm{~km} \mathrm{~s}^{-1}$ \\
Channel width $_{\text {Noise in continuum }}^{\mathrm{a}}$ & & $0.824 \mathrm{~km} \mathrm{~s}^{-1}$ \\
Noise per channel $^{\mathrm{a}}$ & & $0.3 \mathrm{~K}$ \\
$T_{b} / S_{\nu}$ & $2 \mathrm{~K}$ \\
& & $168 \mathrm{~K} / \mathrm{Jy}$ \\
\hline
\end{tabular}

${ }^{a}$ Noise levels may be different from these representative values depending on location and velocity ( $\mathrm{H}$ I line). 
Table 2. Fits of Equation (2)

\begin{tabular}{ccccccc}
\hline \hline Date & $a_{L}$ & $b_{L}$ & $\sigma_{L}$ & $a_{R}$ & $b_{R}$ & $\sigma_{R}$ \\
\hline $07 / 23$ & 1.26 & 1.033 & 0.088 & $\ldots$ & $\ldots$ & $\ldots$ \\
$07 / 25$ & 1.10 & 1.078 & 0.061 & 1.14 & 1.169 & 0.057 \\
$07 / 28$ & 1.28 & 1.079 & 0.060 & 1.32 & 1.182 & 0.061 \\
$07 / 29$ & 1.52 & 1.075 & 0.094 & 1.54 & 1.216 & 0.093 \\
$08 / 02$ & 1.37 & 1.021 & 0.052 & 1.41 & 1.135 & 0.049 \\
$08 / 04$ & 1.38 & 1.133 & 0.055 & 1.44 & 1.276 & 0.051 \\
$08 / 05$ & 1.48 & 1.090 & 0.031 & 1.58 & 1.187 & 0.037 \\
$08 / 08$ & 1.50 & 1.056 & 0.058 & 1.42 & 1.215 & 0.051 \\
$08 / 10$ & 1.41 & 1.079 & 0.076 & 1.51 & 1.166 & 0.071 \\
$08 / 14$ & 1.31 & 1.121 & 0.061 & 1.31 & 1.246 & 0.059 \\
$08 / 17$ & 1.46 & 1.102 & 0.063 & 1.55 & 1.231 & 0.066 \\
$08 / 19$ & 1.30 & 1.055 & 0.026 & 1.31 & 1.141 & 0.029 \\
$08 / 22$ & 1.26 & 1.166 & 0.055 & 1.28 & 1.228 & 0.054 \\
$08 / 24$ & 1.42 & 1.110 & 0.051 & 1.45 & 1.272 & 0.061 \\
$08 / 29$ & 0.89 & 1.118 & 0.090 & 0.91 & 1.228 & 0.093 \\
$08 / 31$ & 1.41 & 1.115 & 0.039 & 1.51 & 1.262 & 0.040 \\
$09 / 05$ & 1.40 & 1.143 & 0.042 & 1.54 & 1.287 & 0.046 \\
$09 / 07$ & 1.64 & 1.115 & 0.058 & 1.75 & 1.260 & 0.058 \\
$09 / 11$ & 1.39 & 1.132 & 0.034 & 1.54 & 1.272 & 0.034 \\
$09 / 14$ & 1.17 & 0.933 & 0.097 & 1.18 & 1.243 & 0.109 \\
$09 / 15$ & 1.41 & 1.147 & 0.066 & 1.42 & 1.314 & 0.061 \\
$09 / 17$ & 1.29 & 1.011 & 0.041 & 1.40 & 1.127 & 0.041 \\
$09 / 18$ & 1.33 & 1.258 & 0.163 & 1.79 & 1.351 & 0.174 \\
$09 / 19$ & 1.32 & 1.139 & 0.065 & 1.46 & 1.284 & 0.061 \\
$09 / 21$ & 1.15 & 1.237 & 0.088 & 1.26 & 1.398 & 0.101 \\
$09 / 24$ & 1.15 & 0.996 & 0.044 & 1.12 & 1.083 & 0.055 \\
$09 / 29$ & 1.34 & 1.146 & 0.054 & 1.51 & 1.233 & 0.050 \\
$09 / 30$ & 1.32 & 1.173 & 0.060 & 1.32 & 1.273 & 0.065 \\
\hline
\end{tabular}


Fig. 1.- (Provided as separate gif image) The Sensitivity Function of the VGPS. A representative section of the primary survey area is shown. Contours are drawn at the $50 \%$, $80 \%, 90 \%, 95 \%$, and $98 \%$ levels of the maximum sensitivity in the mosaic. This function reflects the Gaussian primary beam shape of the VLA and the grid of pointing centers indicated by the crosses. The latitude coverage of the survey widens by two more rows of pointing centers for longitudes greater than $45^{\circ} .7$, and a further two rows for $l>59.7$.

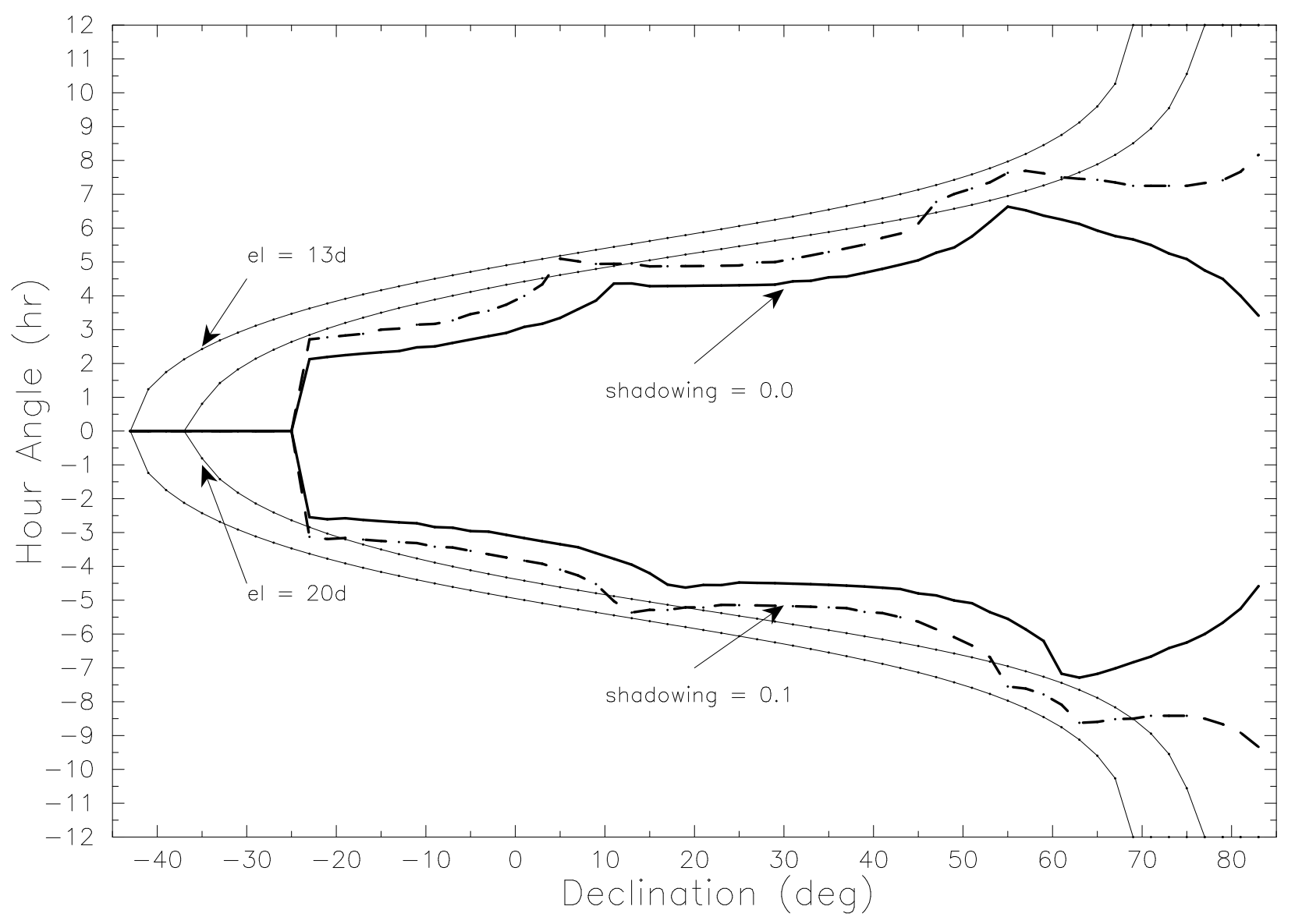

Fig. 2.- Shadowing vs. hour angle and declination for the D array of the VLA. Two elevations, $13^{\circ}$ and $20^{\circ}$, are plotted with thin lines; two shadowing limits, zero shadowing and $10 \%$ shadowing, are plotted with thick lines. These limits were computed using the AIPS subroutines GETANT, UVANT, and BLOCK, for the antenna file corresponding to the array used for all survey observations. All observations of the primary survey area were taken with zero shadowing, i.e., for hour angles in the area between the solid curves. Some of the observations of the higher latitude fields $\left(\left(|b|>1^{\circ}\right)\right.$ were taken with some shadowing, but never more than a few percent. 


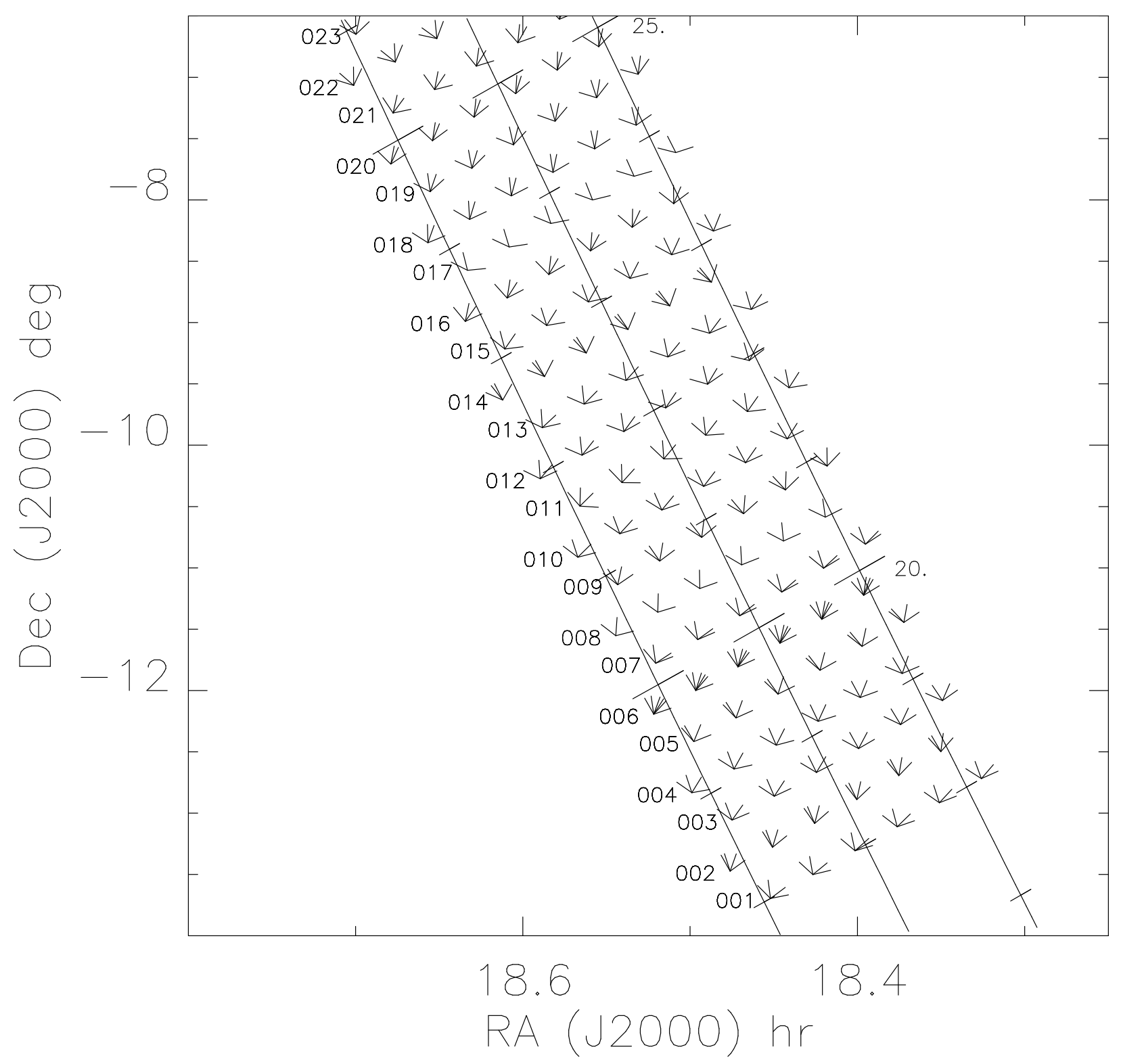

Fig. 3.- Hour angles of the observations of sample pointings. The Galactic plane follows the center line, and the two lines on either side show latitudes $-1^{\circ}$ and $+1^{\circ}$. Longitudes are marked with short bars, with longitude $20^{\circ}$ and $25^{\circ}$ indicated. The pointing centers are shown as the vertices of several short line segments ("chicken feet"). The short segments each represent an observation, with the direction of the segment indicating the corresponding hour angle, with $0^{\mathrm{h}}$ plotted as a vertical segment, $-6^{\mathrm{h}}$ and $+6^{\mathrm{h}}$ plotted horizontally to the left and right. The observing schedule was chosen to optimize the $u v$ coverage provided by a wide range of hour angles, i.e., a broad footprint for each pointing. 


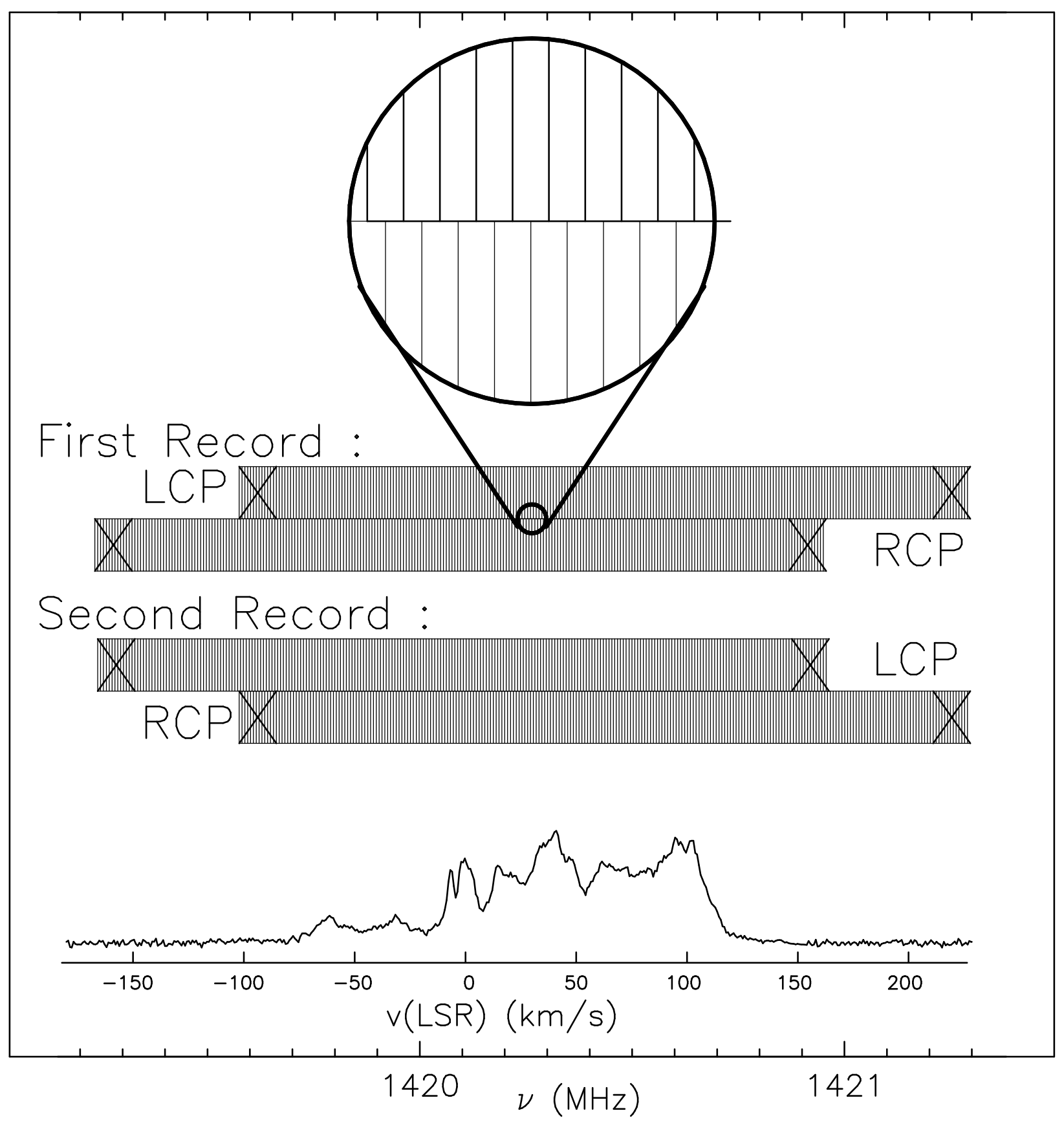

Fig. 4.- Spectrometer channel spacing. Observing frequency is indicated on the bottom, with a representative spectrum shown in the inset, with velocity shifted by a typical offset from the rest frequency of $1420.4058 \mathrm{MHz}$ due to terrestrial and solar motion. All observations were done in pairs, with the center frequencies of the two polarizations switched as indicated. The channel center frequencies are staggered as shown in the magnified inset, so as to make it possible to sample the profile shape with $0.824 \mathrm{~km} \mathrm{~s}^{-1}$ channels in spite of the necessity to take the data with a broader channel spacing $\left(1.28 \mathrm{~km} \mathrm{~s}^{-1}\right)$. The center velocity was set at $v_{c}(l)=+80-(1.6 \times l)$ for each longitude, $l$. 


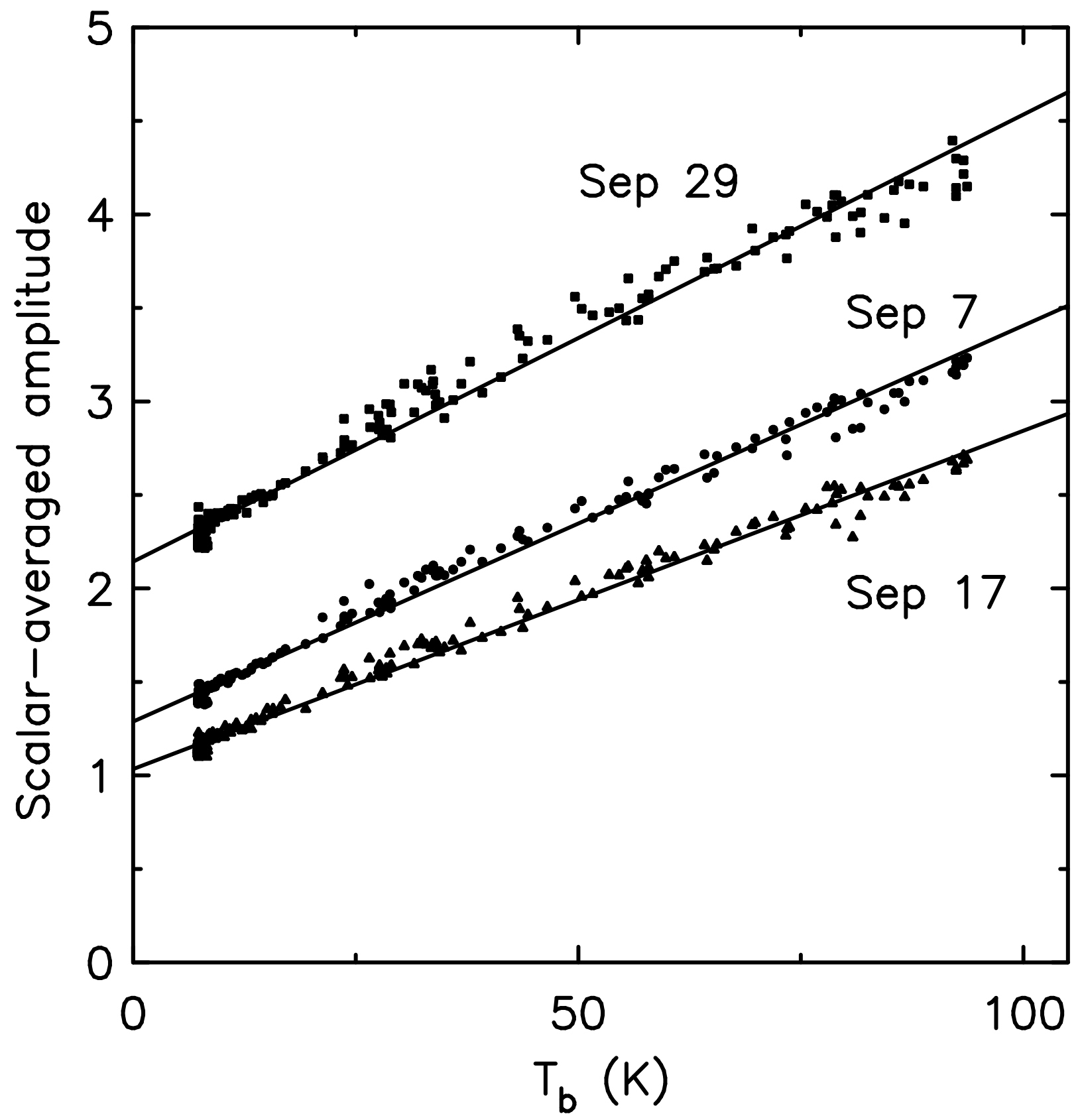

Fig. 5.- Relation between scalar averaged visibility amplitude and sky brightness temperature (line + continuum averaged over the VLA primary beam) for three snapshots of the field G 65.3+1.4 taken with the same spectrometer settings in a single polarization (L) on 2000 September 7, 17, and 29 at elevations 52.3, 63.5, and 24.2 respectively. Each point corresponds to a single frequency channel. The noise per channel changes as the brightness of the $\mathrm{H}$ I line varies with velocity, and a significant variation of the noise level with elevation is apparent. The lines represent linear least squares fits used to separate the contribution of Galactic radio emission from other factors contributing to the noise. 

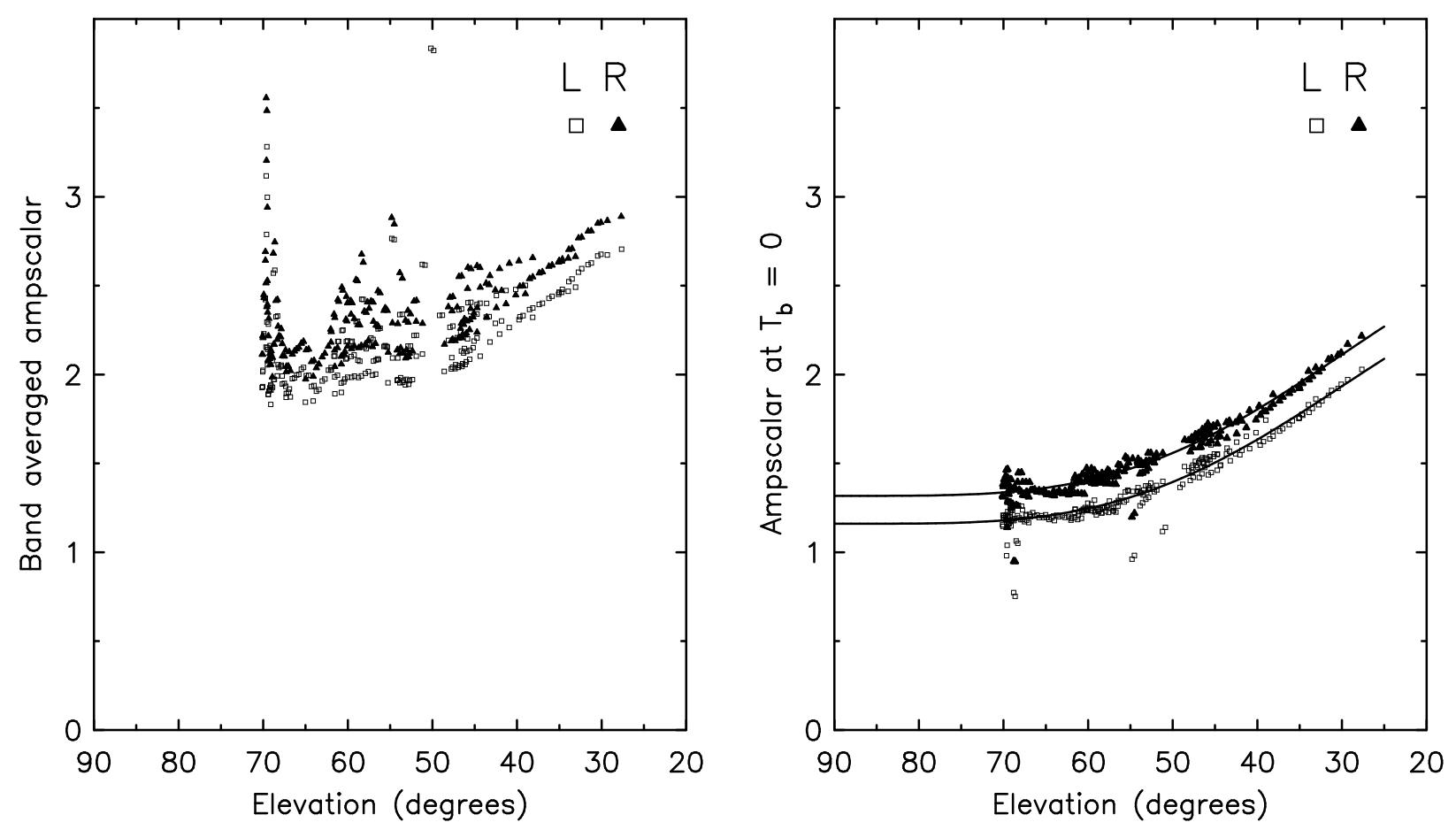

Fig. 6. - Raw band averaged ampscalar (left panel) and ampscalar at $T_{b}=0$ (right panel) as a function of elevation for snapshots taken on 2000 September 15. This figure illustrates the magnitude of contributions to the noise from Galactic H I and continuum emission, and spillover to the ground. Left hand polarization is shown as open squares, right hand polarization as filled triangles. The bright continuum sources W49 and W51 were observed on this day at elevations $50^{\circ}$ and $69^{\circ}$ respectively. The curves in the right panel represent fits of the form $A=a \cos ^{4}(h)+b$, with $h$ the elevation of the field.

Fig. 7.- (Provided as separate gif image) Band averaged ampscalar as a function of position in the sky. Top panel: smallest ampscalar for each field in gray scales. Middle panel: smallest ampscalar per field, corrected for elevation-dependent spillover to the ground. Bottom panel: VGPS continuum image. The predominance of the observing pattern of blocks of 6 fields in the upper panel shows that spillover to the ground is important compared with Galactic emission almost everywhere in the VGPS. When the elevation-dependent contribution is eliminated, as described in the text, a clear correlation with bright Galactic emission is seen. 


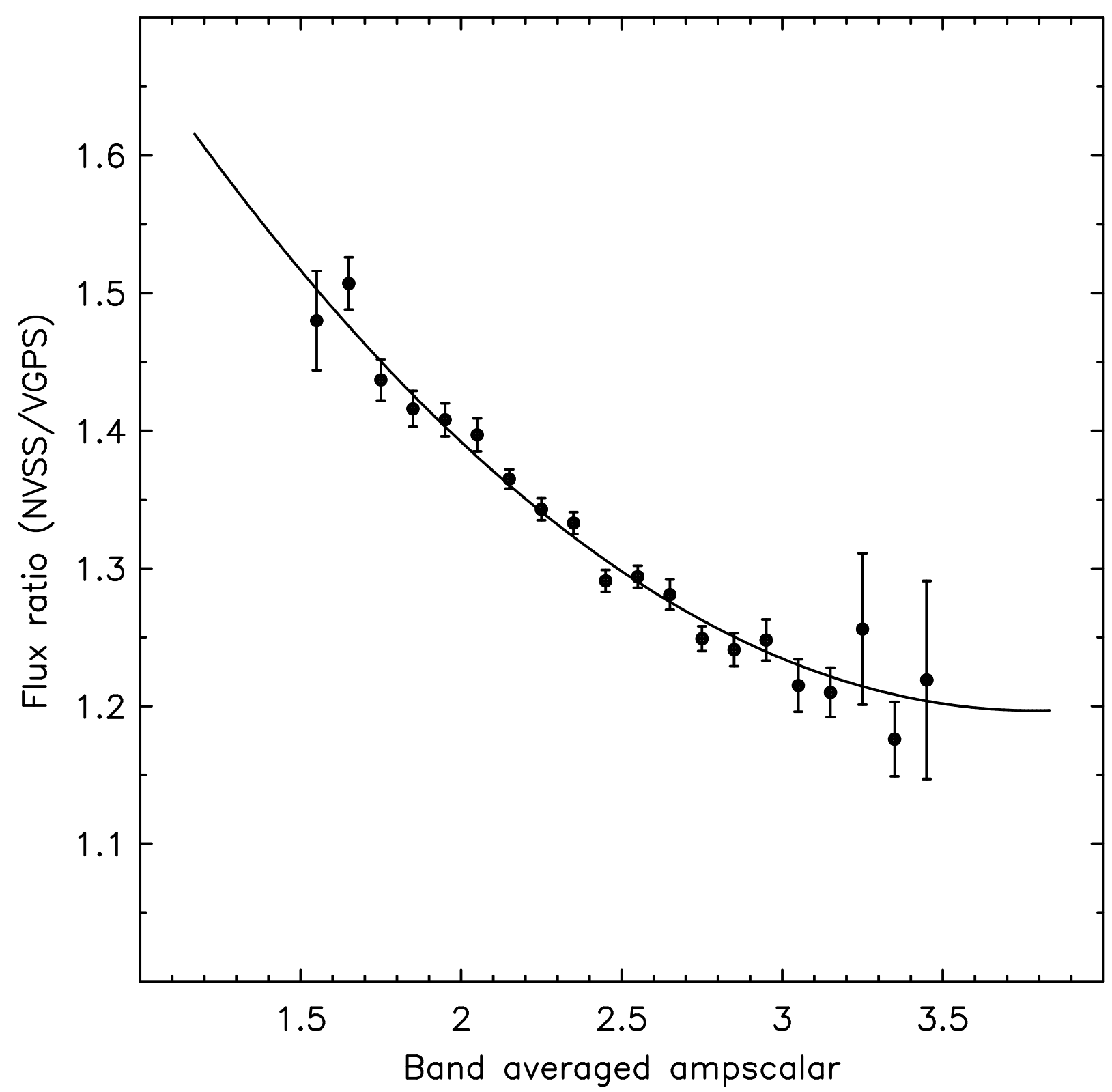

Fig. 8.- The calibrating relation for the VGPS flux correction. The flux ratio (NVSS/VGPS) is shown as a function of band averaged ampscalar, which is proportional to $T_{\text {sys }}$. The solid curve represents the fit that is used to find the appropriate scale factor for individual snapshots. After this correction, the fluxes of compact continuum sources in all VGPS continuum mosaics were found to be consistent with the NVSS to within 5\%. A detailed comparison of the H I spectral line images with the CGPS and SGPS (this paper) further confirms the integrity of the flux scale after this correction. The smaller correction for higher system temperatures is not understood. 
Fig. 9.- (Provided as separate gif image) Sampling of the $u v$ plane and the synthesized beam for a representative field in the center of the survey area. This field is centered on $(l, b)=(42.5,-0.1)$. It was observed three times at hour angles $-2.7,-0.2$, and +2.7 . Left: sampling function of the $u v$ plane. Right: the corresponding synthesized beam, with peak amplitude equal to 1 . Gray scales increase linearly from -0.05 to 0.30 in steps of 0.05 . 

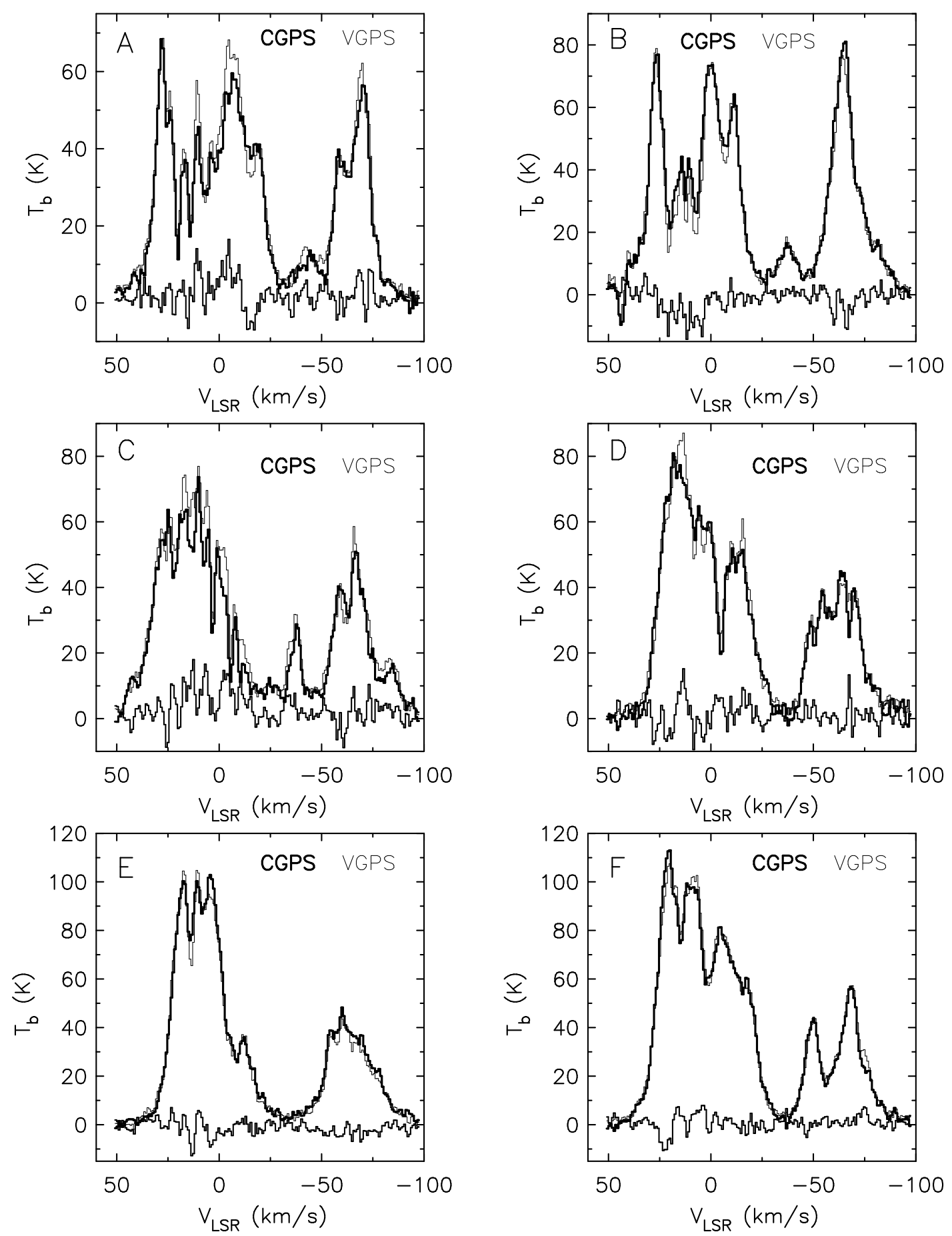

Fig. 10.- Comparison of VGPS and CGPS H I line profiles. Shown are single-position profiles from the CGPS (thick histogram), the VGPS (thin histogram), and the difference (VGPS-CGPS) for six locations. Panels A-D show lines of sight with significant absorption of continuum emission. Panels $\mathrm{E}$ and $\mathrm{F}$ show profiles which are representative for the general field. The theoretical rms of the difference profile is $4.4 \mathrm{~K}$. The surveys show very good agreement, but some systematic differences on the level of 5 to $10 \mathrm{~K}$ are found to persist over several channels. Possible origins of these differences are discussed in Section 3.1. Locations of the profiles in $(l, b)$ are A: $\left(64^{\circ} 13,-00^{\circ} 4\right)$, B: $\left(63^{\circ} 16,+0.45\right), \mathrm{C}:\left(63^{\circ} .00,+0.81\right)$, D: (65.31,-0.22), E: (66.24,-0.31), F: (64.94,-0.27). 
Fig. 11.- (Provided as separate gif image) Difference image (VGPS-CGPS) in the overlap area of VGPS and CGPS phase II for a single velocity channel at $+9 \mathrm{~km} \mathrm{~s}^{-1}$. Black areas on the right are outside the CGPS phase II survey area. The gray scale is linear from $-10 \mathrm{~K}$ to $+10 \mathrm{~K}$. Crosses mark the CGPS field centers. The directions of increasing right ascension and declination are indicated by arrows. The pattern of diagonal dark and light bands aligned with the CGPS field centers originates from the DRAO synthesis telescope. Some artifacts in the VGPS are visible at latitudes $\pm 2^{\circ}$. 

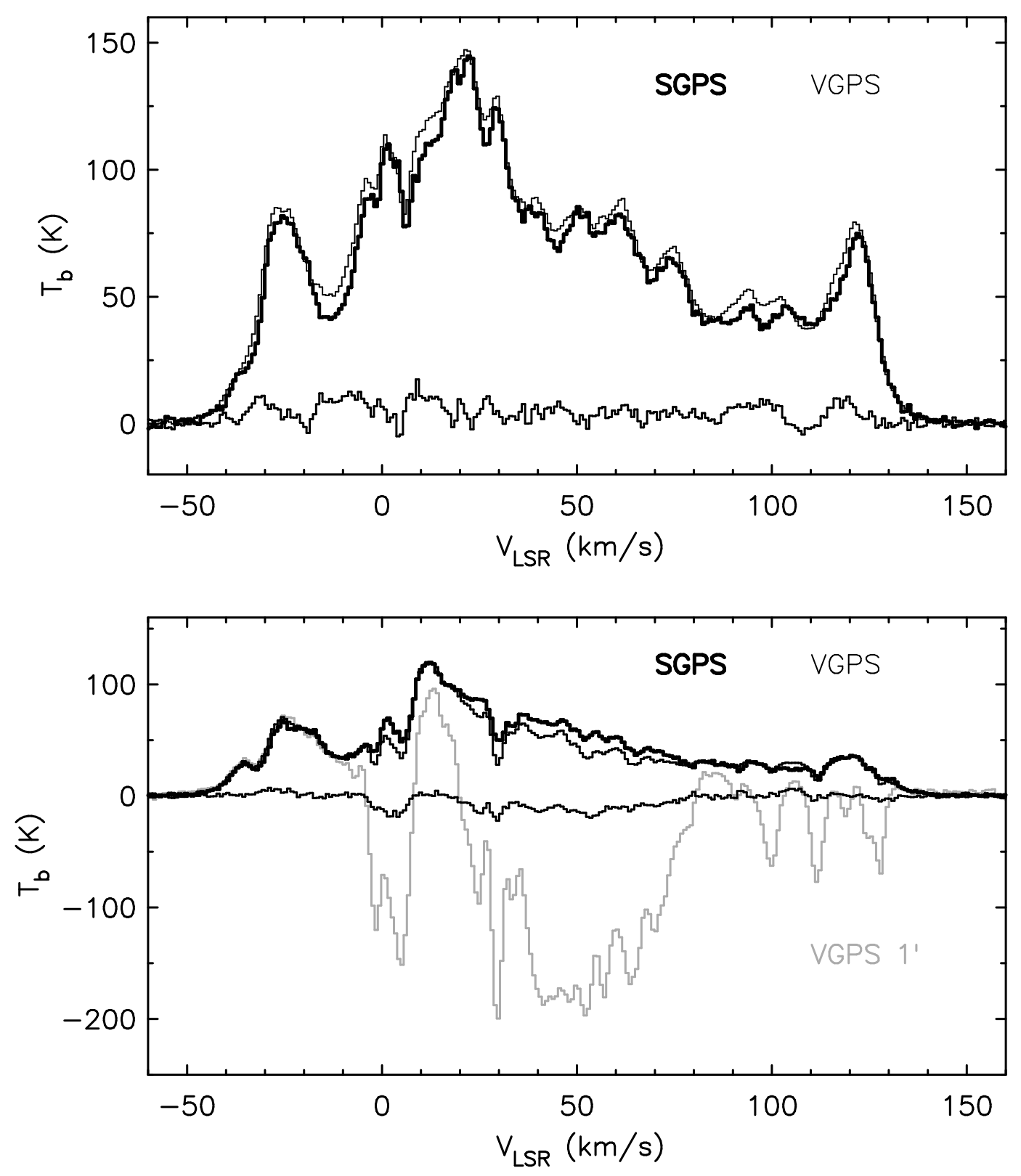

Fig. 12.- Comparison of VGPS and SGPS H I line profiles. The overlap region of the VGPS with the SGPS is the most difficult part of the Galactic plane to observe for both surveys. Shown are single-position profiles from the SGPS (thick histogram), the VGPS convolved to the $3^{\prime} \times 2^{\prime}$ beam of the SGPS (thin histogram), and the difference (VGPS-SGPS) for two locations. The top panel shows a representative H i emission profile at $(l, b)=(19.19,+0.07)$. The bottom panel shows the strongest absorption profile in the overlap area at $(l, b)=$ $(19.61,-0.23)$. The gray profile in the bottom panel is the full-resolution ( $1^{\prime}$ FWHM) VGPS absorption profile at the same location, illustrating that a small discrepancy in the beam size may contribute significantly to the residuals. 
Fig. 13.- (Provided as separate gif image) VGPS panoramic image of the Galactic plane. Top panel: Continuum image. Gray scales are logarithmic from 0 to $90 \mathrm{~K}$. Bottom: $\mathrm{H}$ I spectral line image at velocity $+3.5 \mathrm{~km} \mathrm{~s}^{-1}$. Gray scales are linear from 0 to $125 \mathrm{~K}$. The extent of the spectral line images is limited by the coverage of the GBT data. The continuum images display the Effelsberg data only outside the area covered by the VLA data (see Table 1).

Fig. 13.- (Provided as separate gif image) Continued.

Fig. 13.- (Provided as separate gif image) Continued.

Fig. 13.- (Provided as separate gif image) Continued. 
This figure "f1.gif" is available in "gif" format from: http://arxiv.org/ps/astro-ph/0605422v1 
This figure "f7.gif" is available in "gif" format from: http://arxiv.org/ps/astro-ph/0605422v1 
This figure "f9.gif" is available in "gif" format from: http://arxiv.org/ps/astro-ph/0605422v1 
This figure "f11.gif" is available in "gif" format from: http://arxiv.org/ps/astro-ph/0605422v1 
This figure "f13a.gif" is available in "gif" format from: http://arxiv.org/ps/astro-ph/0605422v1 
This figure "f13b.gif" is available in "gif" format from: http://arxiv.org/ps/astro-ph/0605422v1 
This figure "f13c.gif" is available in "gif" format from: http://arxiv.org/ps/astro-ph/0605422v1 
This figure "f13d.gif" is available in "gif" format from: http://arxiv.org/ps/astro-ph/0605422v1 